\title{
Multimodel Robust Control of Nonlinear Plants: A Case Study
}

\author{
R. H. Nyström, K. V. Sandström, T. K. Gustafsson and H. T. Toivonen \\ Department of Chemical Engineering \\ Åbo Akademi University \\ FIN-20500 Åbo (Turku), FINLAND.
}

Fax: Intl+358-2-2654479

\begin{abstract}
A multimodel controller design procedure combined with gain scheduling methods is evaluated for a highly nonlinear chemical process. The controller synthesis method is based on a mixed $\mathrm{H}_{2} / H_{\infty}$ problem to achieve good quadratic performance and robustness for a multimodel plant description. The performance obtained with the optimal multimodel controller, gain scheduled controllers and conventional PID control are compared and tested on a simulated $\mathrm{pH}$ neutralization process.
\end{abstract}

\section{Introduction}

In many control problems it is physically well motivated to base the design on the minimization of a quadratic cost function. Optimal LQ $\left(\mathrm{H}_{2}\right)$ control theory can be applied to design controllers which minimize quadratic cost functions. Another essential feature in all feedback control is the ability to deal with uncertainties. Standard LQ optimal control theory does not explicitly address robustness issues, and it is well known that optimal LQ controllers may be sensitive to process uncertainties. While the $H_{2}$ norm is related to the performance in terms of quadratic costs, a quantitative characterization of the robustness against norm-bounded process uncertainties is provided by the $H_{\infty}$ norm.

The need to design controllers which achieve good quadratic performance while at the same time satisfying specified robustness criteria has motivated the study of design procedures which combine an $\mathrm{H}_{2}$ cost for optimal performance with an $H_{\infty}$-norm bound to achieve robustness. In particular, various mixed $\mathrm{H}_{2} / H_{\infty}$ control problems have been introduced (Bernstein and Haddad 1989, 
Khargonekar and Rotea 1991, Ridgely et al. 1992a, 1992b, Pensar and Toivonen 1994). A particular feature of the mixed $H_{2} / H_{\infty}$ problems is that they lack closed form solutions, and must therefore be solved by various numerical optimization techniques.

The $H_{\infty}$ norm is related to robustness against unstructured norm-bounded uncertainties. In many cases the uncertainties are known to be structured and/or parametric, and a characterization based on the $H_{\infty}$ norm then leads to a conservative design. Robustness with respect to structured norm-bounded uncertainties can be represented in terms of the structured singular value (Doyle 1982, Doyle et al. 1982, Maciejowski 1989, Skogestad and Postlethwaite 1996). In this approach the uncertainty is described in terms of a structured uncertainty block. Worst-case $\left(H_{\infty}\right)$ performance and structured uncertainties can be treated in a unified framework using the $\mu$-synthesis technique (Doyle et al. 1982, Maciejowski 1989, Skogestad and Postlethwaite 1996). It is, however, very hard to design an $\mathrm{H}_{2}$-optimal controller which achieves robustness against structured uncertainties described by a structured uncertainty block. Also, the available information about process uncertainties is often in a form which is not easy to represent using a structured uncertainty block. For these reasons, it is worth while to study other characterizations of structured uncertainties as well.

An alternative characterization of structured uncertainties is to represent the plant by a discrete set of linear time-invariant models (MacMartin et al. 1991, Mäkilä 1991). The discrete models could describe parametric process uncertainties, or they could represent linearized models of a nonlinear plant at a number of operating points. Such multimodel characterizations have been applied successfully in flight control (Gangsaas et al. 1986, Miyazawa 1990) in order to achieve robustness against model uncertainties and plant variations, and their usefulness has also been demonstrated for chemical process control problems (Sandelin et al. 1991, Toivonen and Tamminen 1990).

Multimodel techniques based on a discrete set of linear models describe the process dynamics in terms of a finite number of linear models, and they are best suited for describing parametric uncertainties. Although the model set can be made arbitrarily large by increasing the number of models, it is not well suited for describing uncertainties which are characterized as norm-bounded operators. A more satisfactory approach, which can be applied to represent uncertain plants with both parametric and norm-bounded uncertainties, is to equip the models in the discrete set with norm-bounded, frequency-weighted unstructured uncertainties (Pensar and Toivonen 1994, Pensar 1996). This 
uncertainty description is quite general, and a large class of structured uncertainties can be represented in this way to any degree of accuracy by selecting a sufficiently large number of models in the discrete set. It is also fairly straightforward to characterize a specified process uncertainty using a multimodel representation.

In this paper, a multimodel mixed $H_{2} / H_{\infty}$ problem is studied and evaluated for a strongly nonlinear $\mathrm{pH}$ neutralization process (Gustafsson et al. 1995). The controller synthesis method is a continuous-time counterpart of a discrete-time problem studied by Pensar and Toivonen (1994) and Pensar (1996). The design procedure is based on a multimodel representation of the process, consisting of a set of linear models and their associated frequency-weighted, norm-bounded uncertainties. An $\mathrm{H}_{2}$-optimal controller is calculated for the set of models subject to robust stability. The model representation is determined so as to characterize the process dynamics in the whole operating region, including appropriate modeling uncertainties.

Due to the strong nonlinearities of the process, it is also well motivated to study control laws in which optimal linear controllers are applied in combination with various controller scheduling methods. Two types of scheduling methods are studied in this paper. The first approach is based on the fact that a major part of the process nonlinearity stems from variations of the stationary gain. Therefore, it appears well motivated to apply variable-gain control to the process. A variable-gain, multimodel mixed $H_{2} / H_{\infty}$-optimal controller can be constructed by designing an optimal linear controller for a multimodel plant representation in which all models have their stationary gains scaled to the same value. The controller is then implemented on the actual plant by applying standard gain scheduling based on known variations of the stationary gain. An appealing feature of this approach is that the controller performance is improved by straightforward gain scheduling, which can easily be taken into account in the controller design and implementation. The second approach studied in this paper is full controller scheduling based on optimal controllers calculated for various operating points. Although this technique gives better performance, it leads to a more complex implementation.

The paper is organized as follows. In Section 2 the multimodel control problem is stated. The multimodel mixed $H_{2} / H_{\infty}$ problem and its solution are presented in Section 3. In Section 4, the control problem is formulated for a $\mathrm{pH}$ neutralization process, and the results achieved with the various controller implementation procedures are presented in Section 5. 


\section{Problem formulation}

In this section a multimodel characterization of uncertain plants and an associated robust $\mathrm{H}_{2}$-optimal control problem is introduced.

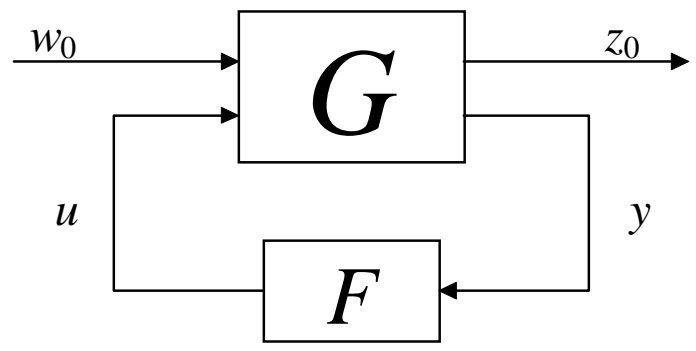

Figure 1. System description.

Consider an uncertain plant $G$ (Figure 1),

$$
\left[\begin{array}{c}
z_{0} \\
y
\end{array}\right]=G\left[\begin{array}{c}
w_{0} \\
u
\end{array}\right], G \in \mathcal{G}
$$

where $z_{0}$ and $y$ are the controlled and measured outputs, respectively, $w_{0}$ is a disturbance, and $u$ is the control signal. The plant is assumed to belong to an uncertainty set $\mathcal{G}$ which is defined by a multimodel description according to

$$
\mathcal{G}:=\bigcup_{k=1}^{N} \mathcal{G}_{k}
$$

where

$$
\mathcal{G}_{k}:=\left\{G_{k}+\left[\begin{array}{c}
0 \\
W_{1 k}
\end{array}\right] \Delta_{k}\left[\begin{array}{ll}
0 & W_{2 k}
\end{array}\right] \mid \Delta_{k}: L_{2} \rightarrow L_{2},\left\|\Delta_{k}\right\| \leq \delta_{k}\right\}, \quad k=1, \ldots N .
$$

Here $G_{k}$ denote finite-dimensional, linear models, and $W_{1 k}$ and $W_{2 k}$ are rational frequency weights for the uncertainties associated with the models. The uncertainty blocks $\Delta_{k}$ are assumed norm-bounded with the uncertainty radii $\delta_{k}$, and they may be nonlinear. The uncertainty description in (2) and (3) provides a characterization of structured plant uncertainties, which combines parametric uncertainties as described by the discrete model set $\left\{G_{k}\right\}$ and norm-bounded uncertainties. 
The control performance is assumed to be specified in terms of a quadratic LQtype cost of the controlled output $z_{0}$ of the uncertain plant (1). The objective is, thus, to minimize the quadratic cost

$$
J(G, F):=\left\|T_{z_{0} w_{0}}(G, F)\right\|_{2}^{2}
$$

where $T_{z_{0} w_{0}}(G, F)$ denotes the closed-loop transfer function in Figure 1 from $w_{0}$ to $z_{0}$ and $\|\cdot\|_{2}$ is the $H_{2}$ norm. In order to design an optimal controller with robustness properties, a robust $H_{2}$-optimal multimodel control problem for the plant (1) is defined as follows.

Problem P1. (Multimodel robust $\mathrm{H}_{2}$-optimal control problem.) Find a linear, time-invariant controller $F$ which minimizes the quadratic cost

$$
J(G, F):=\sum_{k=1}^{N} c_{k} J\left(G_{k}, F\right)
$$

subject to the condition that $F$ stabilizes all $G \in \mathcal{G}$.

In (5), $c_{k}$ are non-negative weights which reflect the importance of the models $G_{k}$ in the quadratic cost. Notice that by minimizing a linear combination of the $H_{2}$ costs for the discrete set $\left\{G_{k}\right\}$, robust $H_{2}$ performance can be achieved with respect to this set, whereas only robust stability is imposed with respect to the norm-bounded uncertainties associated with the models $G_{k}$.

In order to solve Problem P1, it is convenient to pose it as an equivalent multimodel mixed $H_{2} / H_{\infty}$ problem. Consider the process interconnection depicted in Figure 2,

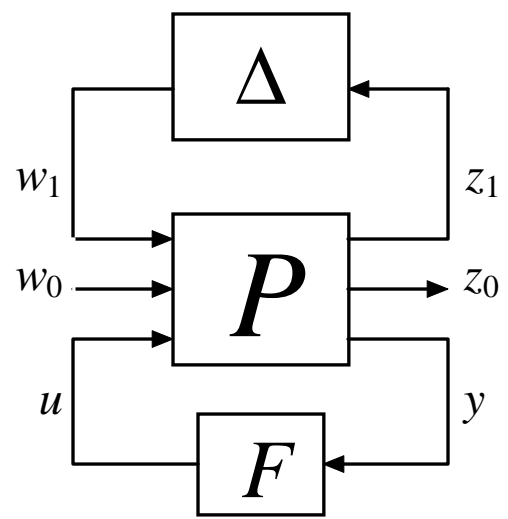

Figure 2. $H_{2} / H_{\infty}$ system description. 
where

$$
\left.P:=\left[\begin{array}{c}
0 \\
0 \\
W_{1}
\end{array}\right] \quad \begin{array}{ll}
0 & W_{2}
\end{array}\right] .
$$

Let $P_{k}$ denote the generalized plant in (6) associated with $G_{k}$ and the weight filters $W_{1 k}$ and $W_{2 k}$, and let $T_{z w}(P, F)$ denote the closed-loop transfer function in Figure 2. The closed-loop system is stable for all norm-bounded uncertainty blocks, $\|\Delta\| \leq \delta$, if and only if the nominal closed-loop system is stable and has $H_{\infty}$-norm from $w_{1}$ to $z_{1}$ less than $\delta^{-1}$ (Maciejowski 1989, Vidyasagar 1985). Hence, Problem P1 is equivalent to the following multimodel mixed $H_{2} / H_{\infty}$ problem.

Problem P2. (Multimodel mixed $H_{2} / H_{\infty}$ control problem.) Find a linear, timeinvariant controller $F$ which stabilizes the plants in the set $\mathcal{P}:=\left\{P_{k}, k=1, \ldots, N\right\}$, and which minimizes the quadratic cost

$$
J(\mathcal{P}, F):=\sum_{k=1}^{N} c_{k} J\left(P_{k}, F\right)
$$

subject to the $H_{\infty}$ norm bounds

$$
\delta_{k}\left\|T_{z_{1} w_{1}}\left(P_{k}, F\right)\right\|_{\infty}<1, k=1, \ldots, N
$$

\section{Solution of multimodel mixed $H_{2} / H_{\infty}$ problem}

The multimodel mixed $H_{2} / H_{\infty}$ control problem has no closed-form solution and it must therefore be solved by numerical optimization methods. It is, however, not straightforward to design efficient optimization procedures for the Problem P2 defined in Section 2. A particular difficulty is presented by the $H_{\infty}$ norm bounds (8), which are infinite-dimensional and nondifferentiable. Here a numerical approach to the controller optimization problem will be presented, in which the $H_{\infty}$ norm bounds are handled indirectly via a penalty function approach. 
Let the plant $P$ have the state-space representation

$$
\begin{aligned}
& \dot{x}_{P}=A_{P} x_{P}+B_{P 0} w_{0}+B_{P 1} w_{1}+B_{P 2} u \\
& z_{0}=C_{P 0} x_{P}+D_{P 02} u \\
& z_{1}=C_{P 1} x_{P}+D_{P 12} u \\
& y=C_{P 2} x_{P}+D_{P 20} w_{0}+D_{P 21} w_{1} .
\end{aligned}
$$

It is assumed that the controller $F$ is a dynamic compensator of fixed order,

$$
\begin{aligned}
& \dot{x}_{C}=A_{C} x_{C}+B_{C} y \\
& u=C_{C} x_{C}+D_{C} y .
\end{aligned}
$$

The following assumptions are imposed on the plant and compensator,
(A1) $D_{P 02} D_{C} D_{P 20}=0$
(A2) $D_{P 12} D_{C} D_{P 21}=0$.

Assumption (A1) is necessary in order to have a finite $H_{2}$ cost, and (A2) is a simplifying assumption which results in a significant simplification of the computational formulas. The assumptions can always be satisfied by fixing the direct term $D_{C}$ of the compensator to zero, if required.

Introduce the closed-loop system $T(P, F)$ defined by (9) and (10),

$$
\begin{aligned}
& \dot{x}=\left(A+B_{2} F C_{2}\right) x+\left(B_{0}+B_{2} F D_{20}\right) w_{0}+\left(B_{1}+B_{2} F D_{21}\right) w_{1} \\
& z_{0}=\left(C_{0}+D_{02} F C_{2}\right) x+D_{02} F D_{21} w_{1} \\
& z_{1}=\left(C_{1}+D_{12} F C_{2}\right) x+D_{12} F D_{20} w_{0}
\end{aligned}
$$

where $x:=\left(x_{P}^{\mathrm{T}}, x_{C}^{\mathrm{T}}\right)^{\mathrm{T}}$,

$$
F:=\left[\begin{array}{ll}
A_{C} & B_{C} \\
C_{C} & D_{C}
\end{array}\right]
$$

and 


$$
\begin{aligned}
& A:=\left[\begin{array}{cc}
A_{P} & 0 \\
0 & 0
\end{array}\right], B_{0}:=\left[\begin{array}{c}
B_{P 0} \\
0
\end{array}\right], B_{1}:=\left[\begin{array}{c}
B_{P 1} \\
0
\end{array}\right], B_{2}:=\left[\begin{array}{cc}
0 & B_{P 2} \\
I & 0
\end{array}\right], \\
& C_{0}:=\left[\begin{array}{ll}
C_{P 0} & 0
\end{array}\right], C_{1}:=\left[\begin{array}{ll}
C_{P 1} & 0
\end{array}\right], C_{2}:=\left[\begin{array}{cc}
0 & I \\
C_{P 2} & 0
\end{array}\right], \\
& D_{02}:=\left[\begin{array}{ll}
0 & D_{P 02}
\end{array}\right], D_{12}:=\left[\begin{array}{ll}
0 & D_{P 12}
\end{array}\right], \\
& D_{20}:=\left[\begin{array}{c}
0 \\
D_{P 20}
\end{array}\right], D_{21}:=\left[\begin{array}{c}
0 \\
D_{P 21}
\end{array}\right] .
\end{aligned}
$$

If the closed-loop system (11) is asymptotically stable, the quadratic cost $J_{2}(P, F)$ defined in compliance with (4) is given by

$$
J_{2}(P, F)=\operatorname{tr}\left\{\left(B_{0}+B_{2} F D_{20}\right)^{\mathrm{T}} Q\left(B_{0}+B_{2} F D_{20}\right)\right\}
$$

where $Q$ is the symmetric positive semidefinite solution of the matrix Lyapunov equation

$$
\left(A+B_{2} F C_{2}\right)^{\mathrm{T}} Q+Q\left(A+B_{2} F C_{2}\right)+\left(C_{0}+D_{02} F C_{2}\right)^{\mathrm{T}}\left(C_{0}+D_{02} F C_{2}\right)=0 .
$$

In order to account for the infinite-dimensional, nondifferentiable $H_{\infty}$ norm bounds (8), an auxiliary cost which serves as a penalty function will be formed. By a standard result in $H_{\infty}$ control theory (Green and Limebeer 1995) the system in (11), assumed to be stable, has $H_{\infty}$ norm from $w_{1}$ to $z_{1}$ less than $\delta^{-1}$ if and only if the Riccati equation

$$
\begin{aligned}
& \left(A+B_{2} F C_{2}\right)^{\mathrm{T}} X+X\left(A+B_{2} F C_{2}\right)+\delta^{2} X\left(B_{1}+B_{2} F D_{21}\right)\left(B_{1}+B_{2} F D_{21}\right)^{\mathrm{T}} X \\
& +\left(C_{1}+D_{12} F C_{2}\right)^{\mathrm{T}}\left(C_{1}+D_{12} F C_{2}\right)=0
\end{aligned}
$$

has a bounded symmetric positive semidefinite solution $X$ such that the matrix

$$
A_{X}:=A+B_{2} F C_{2}+\delta^{2}\left(B_{1}+B_{2} F D_{21}\right)\left(B_{1}+B_{2} F D_{21}\right)^{\mathrm{T}} X
$$

is a stability matrix. This result can be exploited as follows. Introduce the auxiliary cost

$$
J_{\infty}(P, F):=\operatorname{tr}(Z U)
$$


where $Z$ is the solution of the matrix Lyapunov equation

$$
A_{X}^{\mathrm{T}} Z+Z A_{X}+R=0
$$

and where $U$ and $R$ are symmetric positive definite matrices. The auxiliary cost (18) has the property that $J_{\infty}(P, F)$ is bounded if the system $T(P, F)$ in (11) satisfies the $H_{\infty}$ norm bound $\left\|T_{z_{1} w_{1}}(P, F)\right\|_{\infty}<\delta^{-1}$. Moreover, as $\left\|T_{z_{1} w_{1}}(P, F)\right\|_{\infty} \uparrow \delta^{-1}$, the matrix $A_{X}$ in (17) has one or more eigenvalues which approach the stability boundary (the imaginary axis), and $J_{\infty}(P, G) \uparrow \infty$. Hence, the auxiliary cost (18) acts as a penalty function for the $H_{\infty}$ norm bound, and the mixed $H_{2} / H_{\infty}$ problem P2 can be solved to any degree of accuracy via unconstrained problems defined as follows.

Problem P3. (Multimodel mixed $\mathrm{H}_{2} / \mathrm{H}_{\infty}$ problem with penalty function.) Find a linear, time-invariant controller $F$ which stabilizes the plants $P_{k}, k=1, \ldots, N$ and which minimizes the cost

$$
J_{\text {aux }}(\mathcal{P}, F):=\sum_{k=1}^{N}\left[c_{k} J_{2}\left(P_{k}, F\right)+\alpha J_{\infty}\left(P_{k}, F\right)\right]
$$

Here, $\alpha$ is a positive parameter which determines the amount of penalty. From the properties of $J_{\infty}(P, F)$ it follows that the $H_{\infty}$ norm bound (8) is not active at the minimum of (20), and the minimization problem can therefore be treated as an unconstrained optimization problem. Problem P2 can be solved to any degree of accuracy by solving the unconstrained Problem P3 by letting $\alpha \downarrow 0$ (cf. Pensar and Toivonen 1993).

For efficient optimization, an explicit expression for the gradient of the auxiliary cost (20) is required. According to standard parametric LQ control theory, the gradient of (14) with respect to the controller parameters is given by (Anderson and Moore 1971)

$$
\begin{aligned}
\frac{\partial J_{2}(P, F)}{\partial F}= & 2\left[B_{2}^{\mathrm{T}} Q\left(B_{0}+B_{2} F D_{20}\right) D_{20}^{\mathrm{T}}+B_{2}^{\mathrm{T}} Q H C_{2}^{\mathrm{T}}\right. \\
& \left.+D_{02}^{\mathrm{T}}\left(C_{0}+D_{02} F C_{2}\right) H C_{2}^{\mathrm{T}}\right]
\end{aligned}
$$

where $H$ is the symmetric positive semidefinite solution of the matrix Lyapunov equation 


$$
\begin{aligned}
& \left(A+B_{2} F C_{2}\right) H+H\left(A+B_{2} F C_{2}\right)^{\mathrm{T}} \\
& +\left(B_{0}+B_{2} F D_{20}\right)\left(B_{0}+B_{2} F D_{20}\right)^{\mathrm{T}}=0 .
\end{aligned}
$$

The gradient of (18) can be derived in a way similar to the discrete-time case (Pensar and Toivonen 1993, Pensar 1996), and it is given by (see Appendix A)

$$
\frac{\partial J_{\infty}(P, F)}{\partial F}=2 B_{2}^{\mathrm{T}} Z W\left(C_{2}^{\mathrm{T}}+2 X N\right)+M\left(Y+Y^{\mathrm{T}}\right) C_{2}^{\mathrm{T}}+B_{2}^{\mathrm{T}} X\left(Y+Y^{\mathrm{T}}\right) X N
$$

where

$$
\begin{gathered}
M:=B_{2}^{\mathrm{T}} X+D_{12}^{\mathrm{T}}\left(C_{1}+D_{12} F C_{2}\right) \\
N:=\delta^{2}\left(B_{1}+B_{2} F D_{21}\right) D_{21}^{\mathrm{T}}
\end{gathered}
$$

and $W$ and $Y$ are obtained from the Lyapunov equations

$$
\begin{gathered}
A_{X} W+W A_{X}^{\mathrm{T}}+U=0 \\
A_{X} Y+Y A_{X}^{\mathrm{T}}+2 \delta^{2} W Z\left(B_{1}+B_{2} F D_{21}\right)\left(B_{1}+B_{2} F D_{21}\right)^{\mathrm{T}}=0 .
\end{gathered}
$$

\section{A case study: $\mathrm{pH}$ control}

In this section, the controller synthesis method described in Section 3 is applied and evaluated by a simulation study of the $\mathrm{pH}$ neutralization process shown in Figure 3 (Gustafsson et al. 1995).

The process consists of a continuous stirred tank reactor (CSTR) with a constant volume and a constant feed flow resulting in a constant retention time $\tau$, equal to 5 minutes. The effect of the control stream on the retention time is neglected because it is small in comparison to the feed. The feed stream is a water solution of phosphoric acid of varying concentration. The control stream is a concentrated water solution of calcium hydroxide. The control objective is to keep the $\mathrm{pH}$ value of the effluent at a given setpoint value when the process is subjected to variations in feed concentration. 


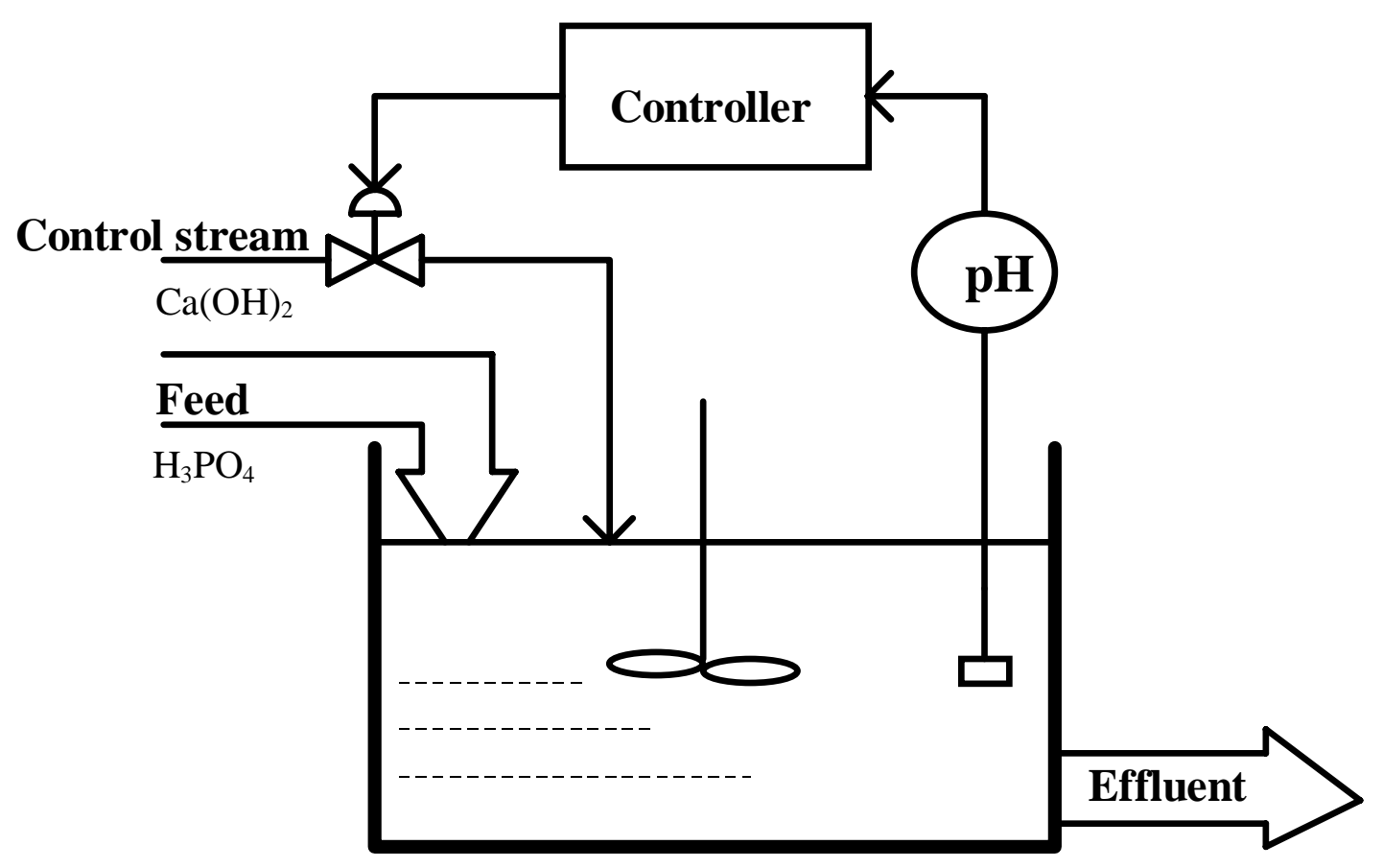

Figure 3. $\mathrm{pH}$ control in a CSTR.

The phosphoric acid of the feed and the calcium hydroxide of the control stream dissociate into the acid-base system $\left\{\mathrm{H}^{+}, \mathrm{H}_{3} \mathrm{PO}_{4}, \mathrm{H}_{2} \mathrm{PO}_{4}^{-}, \mathrm{HPO}_{4}^{2-}\right.$, $\left.\mathrm{PO}_{4}^{3-}, \mathrm{OH}^{-}\right\}$. Acid-base reactions between the ions of this system are fast and in the model they are considered to be at equilibrium at all times. Calcium and phosphate form sparingly-soluble salts, $\mathrm{CaHPO}_{4}$ and $\mathrm{Ca}_{3}\left(\mathrm{PO}_{4}\right)_{2}$, which may precipitate and/or dissolve. The contents and the effluent of the CSTR can thus in addition to the liquid phase also contain a solid phase.

The chemical reactor is modeled with a reaction invariant model (Gustafsson and Waller 1983) augmented with a model of the mass transfer rate between the solid and liquid phases (Gustafsson et al. 1995). The reaction invariant state vector of the liquid phase consists of the concentration of charge (related to the electroneutrality condition) $\left(X_{1}\right)$, the total phosphate concentration $\left(X_{2}\right)$ and the total calcium concentration $\left(X_{3}\right)$. The state of the solid phase consists of the amount of $\mathrm{CaHPO}_{4}$ per reactor volume $\left(X_{4}\right)$ and the amount of $\mathrm{Ca}_{3}\left(\mathrm{PO}_{4}\right)_{2}$ per reactor volume $\left(X_{5}\right)$. The process model is

$$
\begin{aligned}
& \dot{X}(t)=\frac{1}{\tau}\left(X_{f}(t)+X_{u}\left(t-d_{1}\right)-X(t)\right)+r(X(t)) \\
& Y(t)=\varphi\left(X\left(t-d_{2}\right)\right)
\end{aligned}
$$


where $X_{f}$ and $X_{u}$ denote the states of the feed and the control stream, respectively, $Y$ denotes the $\mathrm{pH}$ value of the effluent and $\varphi$ is the nonlinear function which defines the $\mathrm{pH}$ in terms of the states. The state of the feed is $X_{f}=\left[\begin{array}{lllll}0 & W & 0 & 0 & 0\end{array}\right]^{\mathrm{T}}$, where $W$ is the concentration of phosphoric acid. The variation of $W$ is in the sequel considered to be the disturbance to the system. The state of the control stream is $X_{u}=\left[\begin{array}{lllll}0 & 0 & U & 0 & 0\end{array}\right]^{\mathrm{T}}$, where $U$ is defined to be the molar flow of calcium hydroxide divided by feed flow, and it is in the sequel used as control variable.

The pH-measurement system is for simplicity modeled by a time delay, $d_{2}$. Another time delay, $d_{1}$, describes a delay in the control actuator. Both delays have a value of 0.5 minutes.

The mass transfer rate between the liquid and solid states is given by the function $r(X)$, which describes the precipitation and dissolution of solids, and takes the form

$$
r=\left[\begin{array}{c}
0 \\
-r_{a}-2 r_{b} \\
-r_{a}-3 r_{b} \\
r_{a} \\
r_{b}
\end{array}\right]
$$

where $r_{a}$ and $r_{b}$ are the precipitation/dissolution rates of the species $\mathrm{CaHPO}_{4}$ and $\mathrm{Ca}_{3}\left(\mathrm{PO}_{4}\right)_{2}$, respectively. The precipitation/dissolution-rate vector introduces dynamic nonlinearities into the model, contrary to models of fast acid-base reactions which normally involve only static nonlinearities. These rates have been modeled according to a model for crystal growth by Walton (1967), with coefficients partly based on experimental results (Sandström and Gustafsson 1994, Gustafsson et al. 1995). For supersaturated solutions the precipitation rates are given by

$$
\begin{aligned}
& \tilde{r}_{a}=9.68\left(\frac{1}{\mathrm{~mol} \cdot \mathrm{min}}\right) \cdot \tilde{c}_{a}^{2} \\
& \tilde{r}_{b}=156\left(\frac{1^{4}}{\mathrm{~mol}^{4} \cdot \min }\right) \cdot \tilde{c}_{b}^{5}
\end{aligned}
$$


where $\tilde{c}_{a}=\left(10^{3.5}\left(\frac{1}{\mathrm{~mol}}\right) \cdot c_{\mathrm{Ca}^{2+}} \cdot c_{\mathrm{HPO}_{4}^{2-}}-S_{a}^{1 / 2}\right)$ and $\tilde{c}_{b}=\left(10^{20.8}\left(\frac{1}{\mathrm{~mol}}\right)^{4} \cdot c_{\mathrm{Ca}^{2+}}^{3}\right.$ $\left.\cdot c_{\mathrm{PO}_{4}^{3-}}^{2}-S_{b}^{1 / 5}\right)$. Here, the concentrations $c_{\mathrm{Ca}^{2+}}, c_{\mathrm{HPO}_{4}^{2-}}$, etc. are calculated directly from the reaction invariant state, and $S_{a}$ and $S_{b}$ are the solubility products of the respective salts. The rates $r_{a}$ and $r_{b}$ are then given by

$$
\begin{aligned}
& \begin{cases}r_{a}=\tilde{r}_{a}, & \text { if } \tilde{c}_{a}>0 \\
r_{a}=-\tilde{r}_{a}, & \text { if } \tilde{c}_{a}<0 \text { and } X_{4}>0 \\
r_{a}=0, & \text { otherwise }\end{cases} \\
& \begin{cases}r_{b}=\tilde{r}_{b}, & \text { if } \tilde{c}_{b}>0 \text { or }\left(\tilde{c}_{b}<0 \text { and } X_{5}>0\right) \\
r_{b}=0, & \text { otherwise. }\end{cases}
\end{aligned}
$$

The $\mathrm{pH}$ value $Y$ in equation (28) is a nonlinear function $\varphi(\cdot)$ of the reaction invariant states $X_{1}, X_{2}$ and $X_{3}$, and it is defined as the solution of a set of nonlinear equations (Gustafsson et al. 1995). Figure 4 shows an example of the steady-state relation between the control variable $U$ and the $\mathrm{pH}$ of the effluent with a constant feed state at $W=0.01 \mathrm{~mol} / 1$.

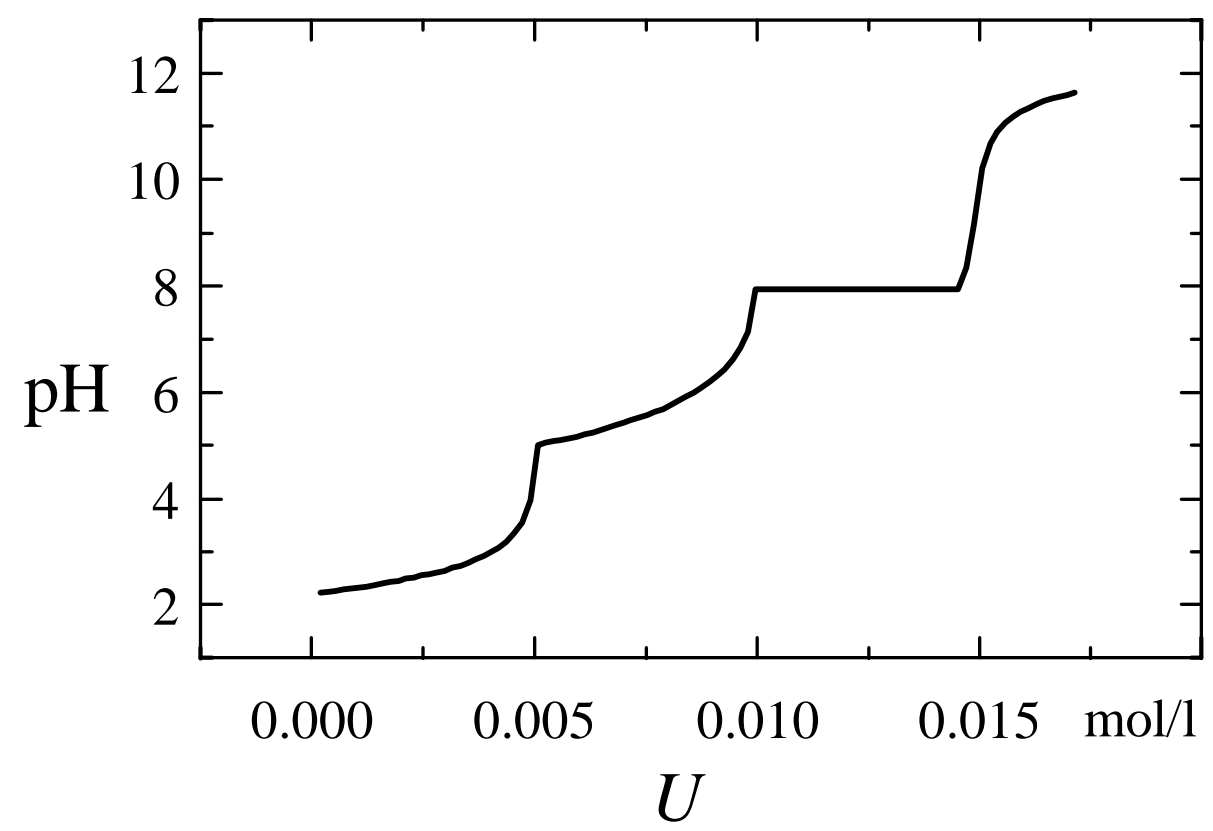

Figure 4. Steady-state relation between control variable $U$ and $\mathrm{pH}$ at $0.01 \mathrm{~mol} / \mathrm{l}$ total concentration of phosphoric acid. 


\subsection{Multimodel description}

The nonlinear system equation (28) can be linearized at a set of stationary points $\left(X_{k}^{s}, Y_{k}^{s}, W_{k}^{s}, U_{k}^{s}\right)$ to give fifth-order linear time delay models. In this particular process the state $X_{1}$ is constant and equal to zero, and the states $X_{4}$ and $X_{5}$ do not affect the states associated with the liquid phase. Therefore, the linearized models can be reduced to second-order time-delay models of the form

$$
\left\{\begin{array}{l}
\dot{x}(t)=A_{k} x(t)+B_{0 k} w(t)+B_{2 k} u\left(t-d_{1}\right) \\
y(t)=C_{2 k} x\left(t-d_{2}\right)
\end{array}\right.
$$

where $x:=X-X_{k}^{s}, y:=Y-Y_{k}^{s}, w:=W-W_{k}^{s}$ and $u:=U-U_{k}^{s}$, and

$$
\begin{aligned}
A_{k} & =\left[\begin{array}{cc}
-\frac{1}{\tau}+\frac{\partial r_{2}}{\partial X_{2}} & \frac{\partial r_{2}}{\partial X_{3}} \\
\frac{\partial r_{3}}{\partial X_{2}} & -\frac{1}{\tau}+\frac{\partial r_{3}}{\partial X_{3}}
\end{array}\right], \quad B_{0 k}=\left[\begin{array}{c}
\frac{1}{\tau} \\
0
\end{array}\right], \quad B_{2 k}=\left[\begin{array}{c}
0 \\
\frac{1}{\tau}
\end{array}\right], \\
C_{2 k} & =\left[\begin{array}{ll}
\frac{\partial \varphi}{\partial X_{2}} & \frac{\partial \varphi}{\partial X_{3}}
\end{array}\right],
\end{aligned}
$$

where the partial derivatives are evaluated at $\left(X_{k}^{s}, Y_{k}^{s}, W_{k}^{s}, U_{k}^{s}\right)$. Due to the complexity of the nonlinear model, the required differentials have been calculated by finite differences. In Table 1 the values of the elements in the matrices $A_{k}$ and $C_{2 k}$ are shown for models at a number of operating points at various $\mathrm{pH}$ values and $W=0.01 \mathrm{~mol} / \mathrm{l}$.

Table 1. The elements of the matrices $A_{k}$ and $C_{2 k}$ at different operating points. The stationary gain is shown in Figure 7.

\begin{tabular}{|c|r|r|r|r|r|r|}
\hline $\mathbf{p H}$ & $\boldsymbol{A}_{\boldsymbol{k}}(\mathbf{1 , 1})$ & $\boldsymbol{A}_{\boldsymbol{k}} \mathbf{( 1 , 2 )}$ & $\boldsymbol{A}_{\boldsymbol{k}} \mathbf{( 2 , 1 )}$ & $\boldsymbol{A}_{\boldsymbol{k}} \mathbf{( 2 , 2 )}$ & $\boldsymbol{C}_{\mathbf{2} \boldsymbol{k}}(\mathbf{1})$ & $\boldsymbol{C}_{\mathbf{2} \boldsymbol{k}} \mathbf{( 2 )}$ \\
\hline 3 & -0.200 & 0 & -0.200 & 0 & -212 & 458 \\
\hline 4 & -0.200 & 0 & -0.200 & 0 & -2068 & 4083 \\
\hline 5 & 0.180 & -0.815 & 0.380 & -1.015 & -2702 & 5457 \\
\hline 6 & 1.024 & -2.634 & 1.224 & -2.834 & -674 & 1197 \\
\hline 7 & 1.306 & -3.890 & 1.580 & -4.202 & -860 & 945 \\
\hline
\end{tabular}

The finite-dimensional linear nominal plant models $G_{k}$ in (3) were constructed by approximating the time-delay operator $\mathrm{e}^{-\left(d_{1}+d_{2}\right) s}$ with a second-order Padé 
approximation $P_{d}(s)$. The uncertainty radii $\delta_{k}$ and weights $W_{1 k}$ and $W_{2 k}$ in (3) were chosen in such a way that the uncertainty sets $\mathcal{G}_{k}$ account for the errors due to the Padé approximation as well as a reasonable amount of unstructured uncertainty for each linear model $G_{k}$. The following simple weights were used,

$$
W_{1 k}(s)=\frac{26}{s+25}\left\|E_{k}\right\|_{\infty}, \quad W_{2 k}(s)=1, \quad k=1, \ldots, N,
$$

where $E_{k}$ denotes the error due to the time-delay approximation,

$$
E_{k}(s):=C_{2 k}\left(s I-A_{k}\right)^{-1} B_{2 k}\left[P_{d}(s)-\mathrm{e}^{-\left(d_{1}+d_{2}\right) s}\right] .
$$

With these weights, the nominal radii of the uncertainty blocks in (3) are $\delta_{k}=1$. Simulations showed, however, that this choice may lead to a slightly conservative design, and that closed-loop stability of the nonlinear plant (28) can still be achieved if the design is based on the uncertainty radii $\delta_{k}=0.5$. A further reduction of the uncertainty radius resulted in instability.

\subsection{Specification of cost function and disturbances}

A crucial part of any controller design based on optimal control methods consists of the specification of the cost and the disturbances in a way which reflects the required controller performance. In practice an important requirement which should be imposed on the controller in most process applications is the ability to cancel the effect of load disturbances on the controlled variable, i.e., the closed loop should have zero gain from the disturbance to the output at zero frequency. The incorporation of an integrator in optimal control is commonly achieved by introducing a frequency-dependent weight on the controlled output with infinite weight at zero frequency. The philosophy of this approach is to replace the quadratic cost function consisting of the square of the $L_{2}$-norm of the output by one which also gives weight on the square of the $L_{2}$-norm of the integral of the output.

An alternative approach, which will be adopted here, is to minimize the original quadratic cost under the assumption that the disturbance is a step. Thus, we consider an uncertain plant $\bar{G}$ described by the state-space equations 


$$
\left\{\begin{array}{l}
\dot{x}_{G}=\bar{A}_{G} x_{G}+\bar{B}_{G s} w+\bar{B}_{G 2} u \\
y=\bar{C}_{G 2} x_{G}+\bar{D}_{G 21} w
\end{array}\right.
$$

Here it is assumed that $w$ is a step disturbance introduced at time $t=0$, i.e.,

$$
w(t)= \begin{cases}w_{s}, & \text { if } t \geq 0 \\ 0, & \text { if } t<0\end{cases}
$$

where $w_{s} \in R^{m}$. The control objective is defined by the quadratic cost

$$
J_{2}(\bar{G}, F):=\sum_{i=1}^{m}\left\{\int_{0}^{\infty}\left[y^{\mathrm{T}} C_{y}^{\mathrm{T}} C_{y} y+\dot{u}^{\mathrm{T}} D_{u}^{\mathrm{T}} D_{u} \dot{u}\right] \mathrm{d} t \mid w_{s}=e_{i}\right\}
$$

where $e_{i}$ denotes the $i$ th unit vector in $R^{m}$, and a quadratic function of $\dot{u}$ rather than $u$ is used in order to make the cost finite. The problem defined by (38) and (40) can be represented in the standard form introduced in Section 2 as follows. The disturbance $w$ can be modeled as

$$
\dot{w}(t)=w_{s} \delta(0)=: w_{0}(t)
$$

where $\delta(\cdot)$ denotes Dirac's $\delta$-function. Combining (38) and (41) gives the state-space model $G$,

$$
\begin{aligned}
& \dot{x}=A_{G} x+B_{G 0} w_{0}+B_{G 2} \dot{u} \\
& z_{0}=C_{G 0} x+D_{G 02} \dot{u} \\
& y=C_{G 2} x
\end{aligned}
$$

where $x:=\left[x_{G}^{\mathrm{T}}, y^{\mathrm{T}}\right]^{\mathrm{T}}, z_{0}:=\left[\left(C_{y} y\right)^{\mathrm{T}},\left(D_{u} \dot{u}\right)^{\mathrm{T}}\right]^{\mathrm{T}}$ and

$$
\begin{aligned}
A_{G} & :=\left[\begin{array}{cc}
\bar{A}_{G} & 0 \\
\bar{C}_{G 2} & 0
\end{array}\right], \quad B_{G 0}:=\left[\begin{array}{c}
\bar{B}_{G s} \\
\bar{D}_{G 21}
\end{array}\right], \quad B_{G 2}:=\left[\begin{array}{c}
\bar{B}_{G 2} \\
0
\end{array}\right], \\
C_{G 0} & :=\left[\begin{array}{cc}
0 & C_{y} \\
0 & 0
\end{array}\right], C_{G 2}:=\left[\begin{array}{ll}
0 & I
\end{array}\right], D_{G 02}:=\left[\begin{array}{c}
0 \\
D_{u}
\end{array}\right] .
\end{aligned}
$$


The cost (40) can be expressed in the form (4) in terms of the $H_{2}$-norm of the closed-loop system consisting of the plant $G$ in (42) and the controller $\dot{u}=F y$. A robust optimal controller for step disturbances can, thus, be determined by applying the procedures in Sections 2 and 3 to the plant defined in (42).

\section{Controller implementations and results}

In this section the multimodel robust optimal controller synthesis procedure described in Sections 2 and 3 is applied to the design of controllers for the $\mathrm{pH}$ neutralization process. As the process is strongly nonlinear, procedures for improving the controller performance by controller scheduling methods to account for the nonlinearities are also studied. The following robust optimal controllers are studied: (1) a fixed linear multimodel $\mathrm{H}_{2} / \mathrm{H}_{\infty}$-optimal controller, (2) a variable-gain multimodel $H_{2} / H_{\infty}$-optimal controller, and (3) a scheduled controller based on $\mathrm{H}_{2} / \mathrm{H}_{\infty}$-optimal controllers designed for various operating points. In all cases, the controller order was taken equal to five which is equal to the order of the generalized plant model, including the weight filters (36) and the second-order Padé approximation. The controllers are designed using two different values of the uncertainty radii, $\delta_{k}=1$ and $\delta_{k}=0.5$. When the smaller value is used, the nominal controller performance in terms of the $H_{2^{-}}$ cost is improved at the expense of closed-loop robustness. For comparison, results are also presented when the process is controlled using a PID controller tuned by conventional means.

The control performance was evaluated for step changes in the concentration $W$ of $\mathrm{H}_{3} \mathrm{PO}_{4}$ in the input flow according to

$$
W(t)= \begin{cases}0.010 \mathrm{~mol} / 1, & t<5 \mathrm{~min} \\ 0.011 \mathrm{~mol} / 1, & 5 \mathrm{~min} \leq t<55 \mathrm{~min} \\ 0.010 \mathrm{~mol} / 1, & t \geq 55 \mathrm{~min} .\end{cases}
$$

The controllers were evaluated at five operating points, corresponding to the $\mathrm{pH}$ values $Y_{k}^{s}=3,4,5,6$ and 7 , and the inputs $U_{k}^{s}=4.00,4.91,5.15,7.06$ and $9.30 \mathrm{mmol} / \mathrm{l}$. 


\subsection{Fixed robust optimal controller}

The multimodel mixed $H_{2} / H_{\infty}$ control problem described in Sections 2 and 3 was applied to design a robust optimal controller for the $\mathrm{pH}$ neutralization process. The controller synthesis was based on twelve linearized models in the $\mathrm{pH}$ range $3-7$. The LQ-cost applied in the controller optimization was defined by (40) with $C_{y}=I$ and $D_{u}=0$, and with equal weights $\left(c_{k}=1\right)$ on the individual costs in the averaged cost (7). No weights on the inputs were required in the quadratic cost (40), since the model uncertainties and the associated conditions for robust stability were sufficient to impose a limitation on the magnitude of the input signals.

The parameters of the controller transfer function

$$
u(s)=\frac{g_{1} s^{5}+g_{2} s^{4}+g_{3} s^{3}+g_{4} s^{2}+g_{5} s+g_{6}}{s\left(s^{5}+f_{1} s^{4}+f_{2} s^{3}+f_{3} s^{2}+f_{4} s+f_{5}\right)} y(s)
$$

of the robust optimal controllers designed for two different uncertainty radii are shown in Table 2.

Table 2. Parameters of fixed optimal robust controllers.

\begin{tabular}{|c|c|c|c|c|c|c|}
\hline $\begin{array}{c}\text { Uncertainty } \\
\text { radius } \boldsymbol{\delta}\end{array}$ & $\boldsymbol{g}_{\mathbf{1}}$ & $\boldsymbol{g}_{\mathbf{2}}$ & $\boldsymbol{g}_{\mathbf{3}}$ & $\boldsymbol{g}_{\mathbf{4}}$ & $\boldsymbol{g}_{\mathbf{5}}$ & $\boldsymbol{g}_{\mathbf{6}}$ \\
\hline 1 & $-1.816 \mathrm{e}-3$ & $-7.075 \mathrm{e}-3$ & $-4.881 \mathrm{e}-3$ & $-8.350 \mathrm{e}-4$ & $-5.405 \mathrm{e}-4$ & $-8.335 \mathrm{e}-7$ \\
\hline 0.5 & $-8.468 \mathrm{e}-3$ & $-4.038 \mathrm{e}-2$ & $-9.491 \mathrm{e}-2$ & $-7.755 \mathrm{e}-2$ & $-2.281 \mathrm{e}-2$ & $-1.230 \mathrm{e}-5$ \\
\hline & $\boldsymbol{f}_{\mathbf{1}}$ & $\boldsymbol{f}_{\mathbf{2}}$ & $\boldsymbol{f}_{\mathbf{3}}$ & $\boldsymbol{f}_{\mathbf{4}}$ & $\boldsymbol{f}_{\mathbf{5}}$ & \\
\hline 1 & $3.991 \mathrm{e}-1$ & $1.233 \mathrm{e} 1$ & $8.549 \mathrm{e}-2$ & 1.418 & $2.074 \mathrm{e}-3$ & \\
\hline 0.5 & 3.576 & $2.377 \mathrm{e} 1$ & $7.814 \mathrm{e} 1$ & $3.628 \mathrm{e} 1$ & $2.287 \mathrm{e}-2$ & \\
\hline
\end{tabular}

Figures 5 and 6 show the output $(\mathrm{pH})$ and the corresponding input $(U)$ when the optimal controllers were simulated for the $\mathrm{pH}$ neutralization process at various operating points. 


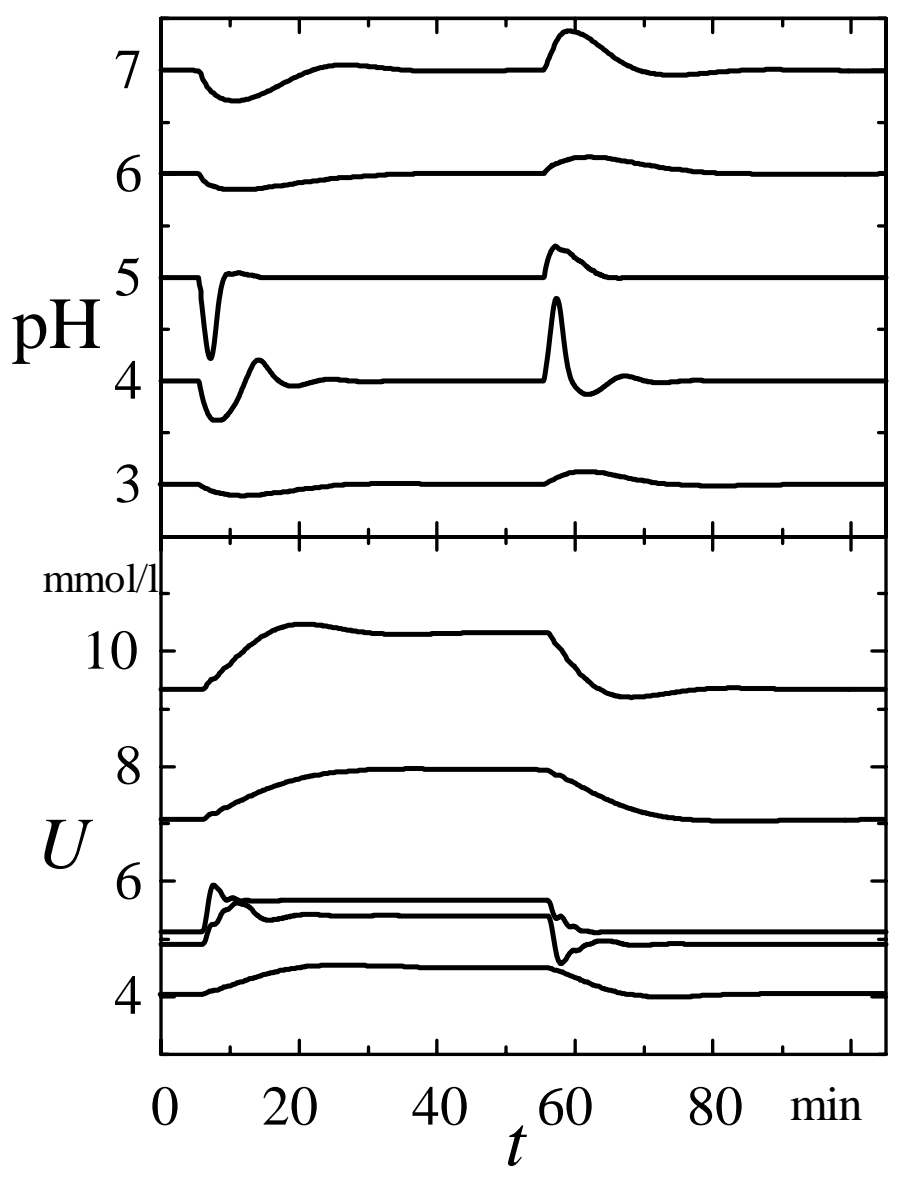

Figure 5. Simulation experiments using fixed robust optimal controller based on uncertainty radius $\delta=1$. 


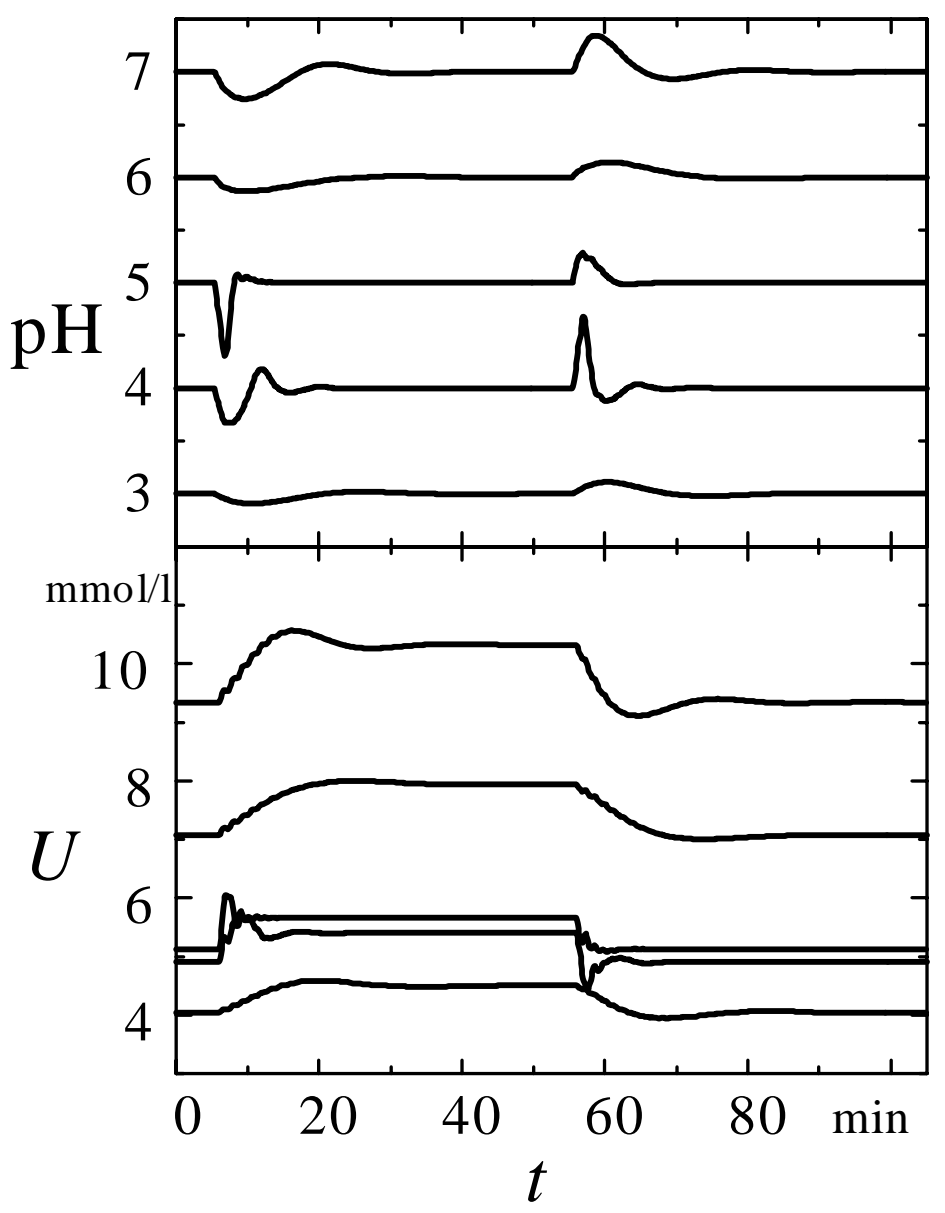

Figure 6. Simulation experiments using fixed robust optimal controller based on uncertainty radius $\delta=0.5$.

The nominal quadratic costs (40) and the $H_{\infty}$-norms for the controllers studied in this paper are summarized in Table 3 at various operating points. The $\mathrm{H}_{2-}$ norms given in the table are the square roots of the quadratic costs defined by (40). 
Table 3. The $H_{2}$-norms and the $H_{\infty}$-norms for the controlled systems using the fixed robust optimal controller, the variable-gain controller (v-gain), the scheduled controller and conventional control (PID) at various operating points.

\begin{tabular}{|l|r|r|r|r|r|}
\hline \multicolumn{1}{|c|}{$\boldsymbol{H}_{\mathbf{2}}$-norm } & $\mathbf{p H}=\mathbf{3}$ & $\mathbf{p H}=\mathbf{4}$ & $\mathbf{p H}=\mathbf{5}$ & $\mathbf{p H}=\mathbf{6}$ & $\mathbf{p H}=\mathbf{7}$ \\
\hline Fixed $(\delta=1)$ & 1725 & 4596 & 3559 & 2463 & 4387 \\
\hline Fixed $(\delta=0.5)$ & 1343 & 3355 & 2687 & 1853 & 3378 \\
\hline V-gain $(\delta=1)$ & 537 & 5239 & 2803 & 586 & 1658 \\
\hline V-gain $(\delta=0.5)$ & 480 & 4682 & 2627 & 538 & 1482 \\
\hline Scheduled $(\delta=1)$ & 237 & 2395 & 2300 & 459 & 810 \\
\hline PID & 2049 & 4464 & 3832 & 3029 & 5146 \\
\hline \multicolumn{1}{|c|}{$\boldsymbol{H}_{\infty}$-norm } & $\mathbf{p H = 3}$ & $\mathbf{p H = 4}$ & $\mathbf{p H = 5}$ & $\mathbf{p H = 6}$ & $\mathbf{p H}=\mathbf{7}$ \\
\hline Fixed $(\delta=1)$ & 0.141 & 0.679 & 0.887 & 0.337 & 0.263 \\
\hline Fixed $(\delta=0.5)$ & 1.220 & 1.487 & 1.794 & 1.705 & 1.489 \\
\hline V-gain $(\delta=1)$ & 0.375 & 0.375 & 0.792 & 0.869 & 0.471 \\
\hline V-gain $(\delta=0.5)$ & 0.508 & 0.508 & 1.359 & 1.299 & 0.633 \\
\hline Scheduled $(\delta=1)$ & 0.947 & 0.991 & 0.965 & 0.940 & 0.984 \\
\hline PID & 0.242 & 1.705 & 2.105 & 0.586 & 0.466 \\
\hline
\end{tabular}

\subsection{Variable-gain optimal robust multimodel controller}

Although the global performance of the multimodel optimal controller is better than that which can be achieved by controllers which are optimized for the various operating points, its performance at the individual stationary points is considerably poorer than the performance which could be achieved by applying locally optimal linear controllers which are optimized at the various stationary points. This can be seen by comparing the nominal costs achieved with the fixed robust optimal controller and the scheduled controller (Section 5.3), cf. Table 3. This is due to the strongly nonlinear dynamics of the process, which limit the performance which can be achieved by any fixed linear control law. A natural approach to improve the global performance of linear controllers is to apply controller scheduling methods. In particular, much of the nonlinearity of the $\mathrm{pH}$ neutralization process is due to large variations of the stationary gain, cf. Figure 7 and Table 1 . Therefore, it is likely that large improvements could be obtained by introducing a controller with scheduling of the stationary gain.

Here a simple variable-gain controller design is introduced, which combines the optimal multimodel controller design procedure with scheduling of the controller gain. In this approach, the effect of gain variations is first eliminated in the controller synthesis by a scaling of the linearized models so that all models have unit stationary gain from the control signal $u$ to the measured 
output $y$, i.e., the nominal models $G_{k}$ and the uncertainty weights $W_{1 k}$ of the sets (3) are scaled according to

$$
G_{k}^{n}:=\left[\begin{array}{cc}
I & 0 \\
0 & K_{k}^{-1}
\end{array}\right] G_{k}, \quad W_{1 k}^{n}:=K_{k}^{-1} W_{1 k} \quad k=1, \ldots, N
$$

where $K_{k}$ denotes the stationary gain from $u$ to $y$ of the nominal model $G_{k}$. The multimodel mixed $H_{2} / H_{\infty}$-optimal controller synthesis problem is then solved for the set of scaled linear models to give an optimal fixed linear controller. When the controller is implemented, the scaling is taken into account by a corresponding rescaling of the controller gain. However, due to the on-line scheduling of the controller gain, the resulting controller is nonlinear, and some caution must therefore be exercised in order to ensure that the closed loop has the desired properties.

In order to develop the nonlinear scheduled control law in a proper way, the dynamics of the resulting closed-loop nonlinear system should be studied. Therefore, suppose that the nonlinear plant is described by

$$
\begin{aligned}
& \dot{X}=f\left(X, W_{0}, U\right) \\
& Z_{0}=g(X, U) \\
& Y=h\left(X, W_{0}\right)
\end{aligned}
$$

and that a number of linearized, uncertain, plant models $G_{k}$ about stationary states $\left(X_{k}^{s}, Y_{k}^{s}, Z_{0 k}^{s}, W_{0 k}^{s}, U_{k}^{s}\right)$ have been determined. Let the scaled models $G_{k}^{n}$ have the state-space representations

$$
\begin{aligned}
& \dot{x}=A_{G k} x+B_{G k 0} w_{0}+B_{G k 2} u \\
& z_{0}=C_{G k 0} x+D_{G k 02} u \\
& y=K_{k}^{-1} C_{G k 2} x+K_{k}^{-1} D_{G k 20} w_{0}, \quad k=1, \ldots, N
\end{aligned}
$$

where $x, u, z_{0}, w_{0}$ and $y$ denote differences from the respective stationary values. Suppose that the optimal multimodel controller $F$ computed for the set of scaled linear plant models and associated uncertainties in (46) is given by

$$
\begin{aligned}
& \dot{x}_{c}=A_{c} x_{c}+B_{c} y \\
& u=C_{c} x_{c}+D_{c} y .
\end{aligned}
$$


To account for scaling, the controller should be scaled by the inverse gain $K_{k}^{-1}$ when applied to the actual plant at the stationary state $\left(X_{k}^{s}, Y_{k}^{s}, Z_{0 k}^{s}, W_{0 k}^{s}, U_{k}^{s}\right)$. As long as the disturbances are small the properly scaled linear controller (49) can be expected to achieve good performance for the nonlinear plant as well. However, for plants which are subject to large disturbances, causing substantial deviations from the stationary point, poor performance may result due to variations of the process gain. A controller which can be applied over the whole operating range can be designed using the linear control law (49) in combination with a gain-scheduling procedure. A variable-gain version of (49) which uses the measured output $Y$ as scheduling variable takes the form

$$
\begin{aligned}
& \dot{x}_{c}=A_{c} x_{c}+B_{c} L(Y)\left(Y-Y_{0}\right) \\
& U=U_{0}+C_{c} x_{c}+D_{c} L(Y)\left(Y-Y_{0}\right), \quad k=1, \ldots, N
\end{aligned}
$$

where $Y_{0}$ denotes a setpoint value for $Y, U_{0}$ is the corresponding stationary input value, and the function $L(Y)$ defines the controller gain as a function of $Y$.

It is important that the scheduling in (50) is designed in such a way that the closed-loop system has acceptable behavior. In this context it is worth while to notice that an interpolation between the linear control laws (49), such that $L\left(Y_{k}^{s}\right)=K_{k}^{-1}$ holds, does not ensure good closed-loop behavior, and may even give rise to instability (Kaminer et al. 1995, Lawrence and Rugh 1995). This is due to the fact that the nonlinear part of the scheduled control law (50) will also affect the closed-loop dynamics. A minimum requirement on a scheduled controller is that when the closed-loop system consisting of the plant (47) and the nonlinear controller (50) is linearized about the stationary states $\left(X_{k}^{s}, Y_{k}^{s}, Z_{0 k}^{s}, W_{0 k}^{s}, U_{0 k}^{s}\right)$ at which the linearized models (48) are valid, it is described by the closed-loop linear system defined by (48), (49). This is the linearization condition discussed in the literature, cf. Kaminer et al. (1995), Lawrence and Rugh (1995). It is straightforward to establish that a sufficient condition for the linearization condition to be satisfied by the controller (50) is given by

$$
\left(\frac{\partial L(Y)}{\partial Y}\right)_{Y=Y_{k}^{s}}\left(Y-Y_{0}\right)+L\left(Y_{k}^{s}\right)=K_{k}^{-1}, \quad k=1, \ldots, N
$$

Notice that under the natural assumption that the setpoint $Y_{0}$ belongs to the set of stationary points $\left\{Y_{k}^{s}\right\}$, e.g. $Y_{0}:=Y_{k_{0}}^{s},(51)$ implies that 


$$
L\left(Y_{0}\right)=K_{k_{0}}^{-1}
$$

At the other steady-state values $Y_{k}^{s}, k \neq k_{0}$ the variable-gain controller (50) is, however, not equal to a scaling of (49) by $K_{k}^{-1}$.

A variable-gain controller which satisfies the linearization criterion (51) at the design points $Y_{1}^{s}<Y_{2}^{s}<\cdots<Y_{N}^{s}$ was constructed for the $\mathrm{pH}$ neutralization process by taking $L(Y)$ as a piecewise quadratic function in the intervals $Y_{k} \leq Y \leq Y_{k+1}, k=1, \ldots, N-1$, with the requirements that it is continuous and satisfies (51) at the design points. These conditions determine the controller scaling function $L(Y)$ uniquely for a given setpoint. Figure 7 shows the resulting function $L(Y)^{-1}$ constructed for the setpoint $Y_{0}=5$. Notice in particular the difference between controller scaling and the stationary gain of the plant.

A variable-gain controller based on the optimal robust multimodel controller design procedure described in Sections 2 and 3 can be constructed by applying the gain-scheduling technique to the linear controller obtained by designing a mixed $H_{2} / H_{\infty}$-optimal controller for the scaled linearized plant models (48). Table 4 gives the parameters of the transfer function representation (45) of linear multimodel $\mathrm{H}_{2} / \mathrm{H}_{\infty}$-optimal controllers designed for the $\mathrm{pH}$ neutralization process for two different uncertainty radii. Table 3 shows the nominal performance of the controllers at various operating points. The responses obtained when the variable-gain controllers are applied to the $\mathrm{pH}$ neutralization process are shown in Figures 8 and 9. As the numerical data in Table 3 indicate, the global control performance obtained with the variable-gain controllers is significantly better than the performance which could be achieved with fixed linear control laws (cf. Figures 5 and 6). Notice, however, that it is important that the scheduling is performed in such a way that the linearization conditions (51) hold. In contrast to the responses shown in Figures 8 and 9, a variable-gain controller based on the inverse of the process gain $K(Y)$ (Figure 7) results in instability of the closed loop for large disturbances. 
Table 4. Parameters of optimal robust multimodel controllers, based on scaled linear models.

\begin{tabular}{|c|c|c|c|c|c|c|}
\hline $\begin{array}{c}\text { Uncertainty } \\
\text { radius } \boldsymbol{\delta}\end{array}$ & $\boldsymbol{g}_{\mathbf{1}}$ & $\boldsymbol{g}_{\mathbf{2}}$ & $\boldsymbol{g}_{\mathbf{3}}$ & $\boldsymbol{g}_{\mathbf{4}}$ & $\boldsymbol{g}_{\mathbf{5}}$ & $\boldsymbol{g}_{\mathbf{6}}$ \\
\hline 1 & $-3.695 \mathrm{e} 1$ & $-5.577 \mathrm{e} 2$ & $-2.730 \mathrm{e} 3$ & $-6.877 \mathrm{e} 3$ & $-7.122 \mathrm{e} 3$ & $-1.603 \mathrm{e} 3$ \\
\hline 0.5 & $-3.157 \mathrm{e} 1$ & $-6.981 \mathrm{e} 2$ & $-5.850 \mathrm{e} 3$ & $-1.735 \mathrm{e} 4$ & $-3.961 \mathrm{e} 4$ & $-1.430 \mathrm{e} 4$ \\
\hline & $\boldsymbol{f}_{\mathbf{1}}$ & $\boldsymbol{f}_{\mathbf{2}}$ & $\boldsymbol{f}_{\mathbf{3}}$ & $\boldsymbol{f}_{\mathbf{4}}$ & $\boldsymbol{f}_{\mathbf{5}}$ & \\
\hline 1 & $1.889 \mathrm{e} 1$ & $1.609 \mathrm{e} 2$ & $9.510 \mathrm{e} 2$ & $3.033 \mathrm{e} 3$ & $1.666 \mathrm{e} 3$ & \\
\hline 0.5 & $2.298 \mathrm{e} 1$ & $2.335 \mathrm{e} 2$ & $1.388 \mathrm{e} 3$ & $5.833 \mathrm{e} 3$ & $1.273 \mathrm{e} 4$ & \\
\hline
\end{tabular}

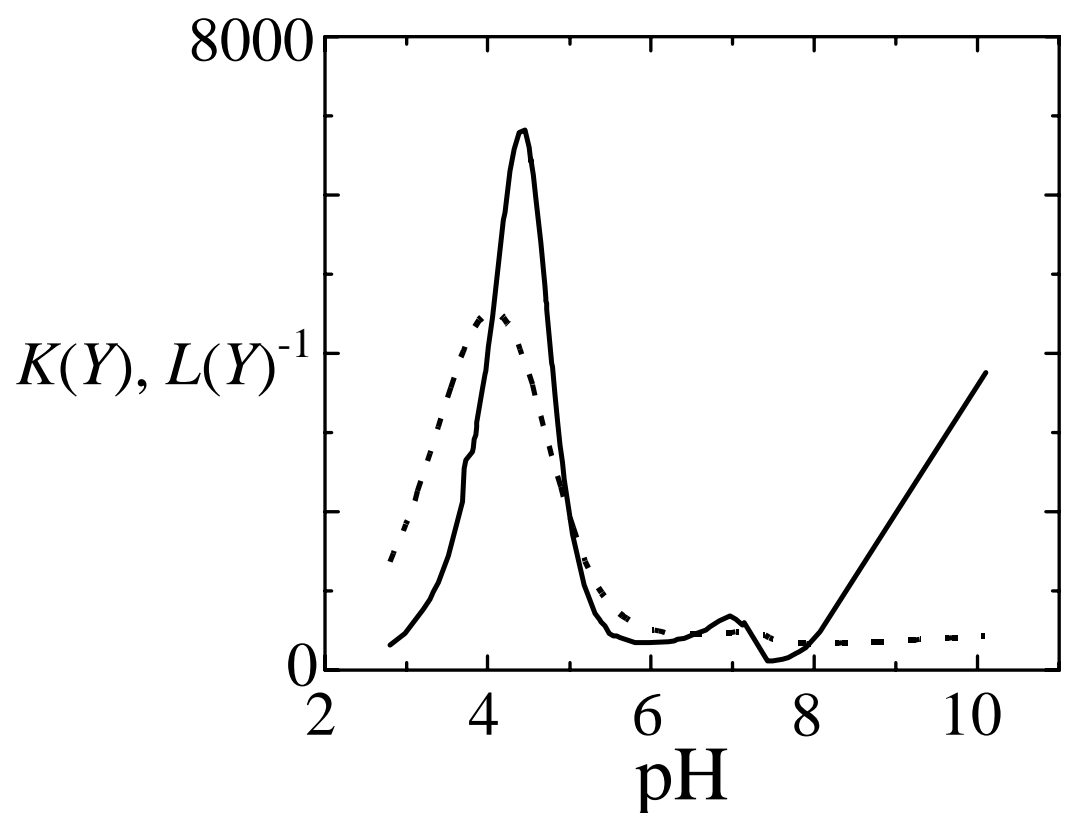

Figure 7. The stationary gain of the process (solid line) and the gain used for variable-gain controller output at setpoint $\mathrm{pH}=5$ (dashed line). 


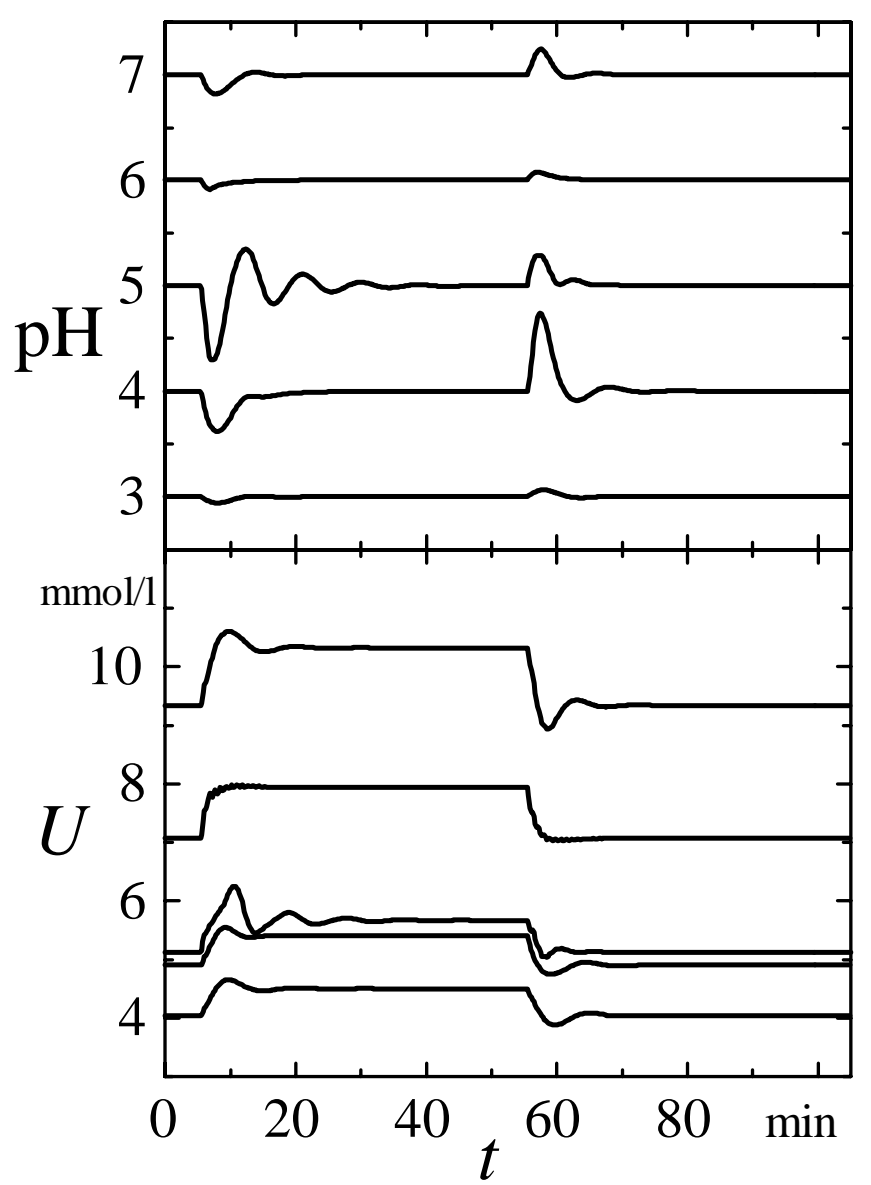

Figure 8. Simulation experiments using variable-gain robust multimodel optimal controller based on uncertainty radius $\delta=1$. 


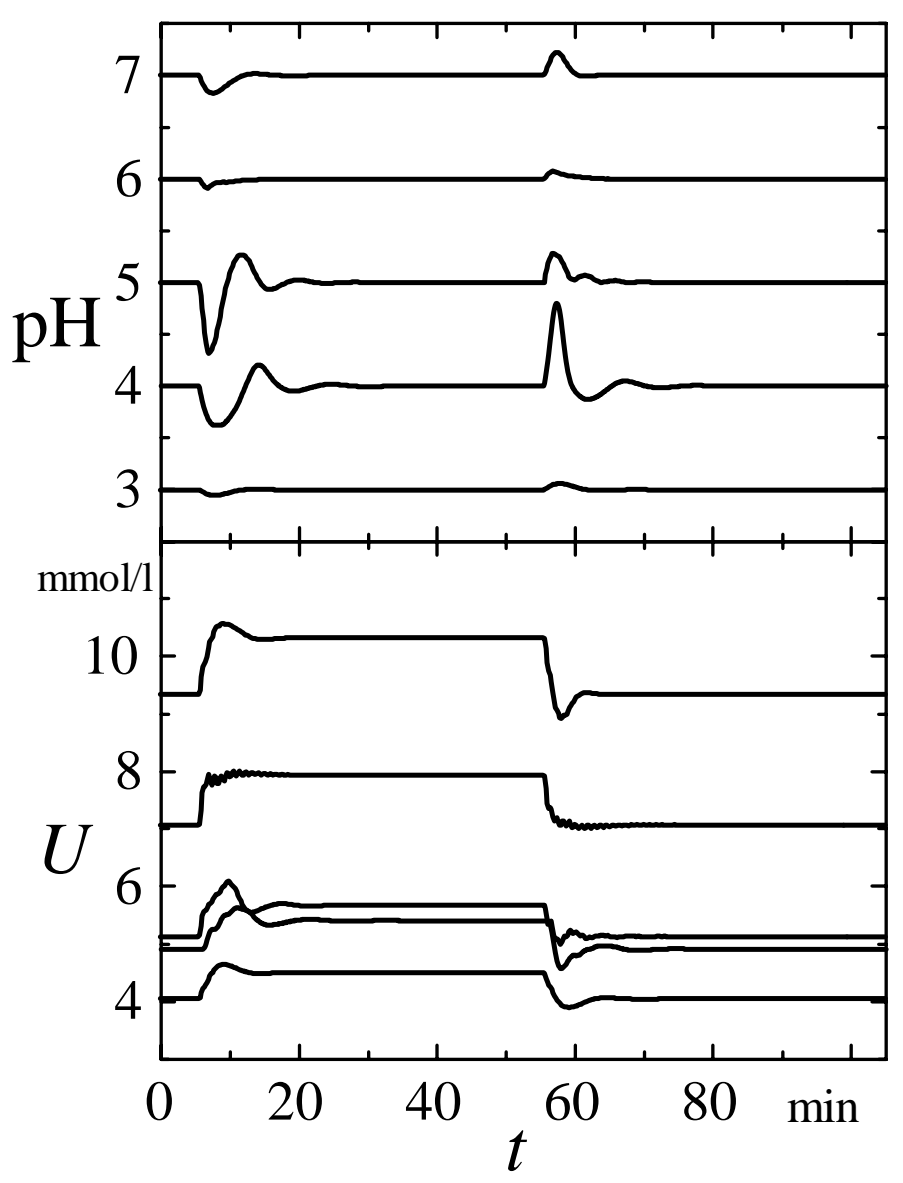

Figure 9. Simulation experiments using variable-gain robust multimodel optimal controller based on uncertainty radius $\delta=0.5$.

\subsection{Scheduled robust optimal control}

In addition to the variable-gain controller described in Section 5.2, it is also of interest to study the performance which is obtained by applying controller scheduling to the locally optimal mixed $H_{2} / H_{\infty}$ controllers valid at the various stationary points. For the $\mathrm{pH}$ neutralization process, it turned out that it is very hard to achieve acceptable control performance by scheduling of the controller parameters using the measured output $Y$ as scheduling parameter. This may be due to the high number of parameters which are adjusted on-line and the fact that the measured $\mathrm{pH}$-value alone may not provide sufficient information for the scheduling of all controller parameters. Instead, a controller scheduling procedure based on the interpolation of the controller transfer functions rather than the parameters of the control law was more successful.

The controller scheduling method applied here can be described as follows. Let $F_{k}, k=1, \ldots, N$ denote the linear optimal control laws designed for the 
linearized plant models at the stationary points $Y_{k}^{s}$, and let $u_{k}$ be the output of the controller $F_{k}$ when the process is controlled at the setpoint $Y_{0}$,

$$
u_{k}:=F_{k}\left(Y-Y_{0}\right)
$$

The scheduled controller is now taken as the linear combination

$$
u(t)=\alpha_{k}(Y) u_{k}(t)+\alpha_{k+1}(Y) u_{k+1}(t), \text { if } Y_{k}^{s} \leq Y(t) \leq Y_{k+1}^{s} .
$$

The linearization conditions for the scheduled controller (54) take the form

$$
\alpha_{k, e f f}\left(Y_{k^{\prime}}^{s}\right)=\left\{\begin{array}{ll}
1, & \text { if } k=k^{\prime} \\
0, & \text { if } k \neq k^{\prime}
\end{array} \quad, \quad k=1, \ldots, N, k^{\prime}=k-1, k, k+1\right.
$$

where

$$
\alpha_{k, e f f}(Y):=\left(\frac{\partial \alpha_{k}(Y)}{\partial Y}\right)\left(Y-Y_{0}\right)+\alpha_{k}(Y)
$$

The quantity in (56) can be regarded as the effective weight on the controller $F_{k}$ in the nonlinear scheduled controller. It is also natural to require that $0 \leq \alpha_{k}(Y) \leq 1$. In order to achieve continuity at the design points $Y_{k}^{s}$ and smooth switching between controllers it is also required that the functions $\alpha_{k}(Y)$ satisfy

$$
\alpha_{k}\left(Y_{k-1}^{s}\right)=\alpha_{k}\left(Y_{k+1}^{s}\right)=0 \quad \text { and } \quad \alpha_{k}\left(Y_{k}^{s}\right)=1
$$

A function which satisfies the conditions (55)-(57) is given by

$$
\begin{aligned}
& \alpha_{k}(Y)=\frac{1}{2} \cos \left(\pi \frac{Y-Y_{k}^{s}}{Y_{k+1}^{s}-Y_{k}^{s}}\right)+\frac{1}{2}, \quad Y_{k}^{s} \leq Y \leq Y_{k+1}^{s} \\
& \alpha_{k}(Y)=-\frac{1}{2} \cos \left(\pi \frac{Y-Y_{k-1}^{s}}{Y_{k}^{s}-Y_{k-1}^{s}}\right)+\frac{1}{2}, \quad Y_{k-1}^{s} \leq Y \leq Y_{k}^{s} .
\end{aligned}
$$

The function (58) has the nice properties that $\alpha_{k}(Y)+\alpha_{k+1}(Y)=1$ and $\alpha_{k, \text { eff }}(Y)+\alpha_{k+1, e f f}(Y)=1$ for all $Y_{k}^{s} \leq Y \leq Y_{k+1}^{s}$. These properties imply that 
both the nominal and the effective weights on the controllers in (54) sum to unity for all $Y$.

A potential problem with the scheduling procedure described here is the possibility of windup due to the fact that the output of the individual controllers are not equal to the actual input to the process. For this reason any common unstable modes of the controllers should be moved outside the switching mechanism.

Table 5 gives the parameters of the transfer function representation (45) of locally optimal mixed $\mathrm{H}_{2} / \mathrm{H}_{\infty}$ controllers for the $\mathrm{pH}$ neutralization process calculated for various operating points. The uncertainty radius used in the controller design was $\delta=1$. The nominal performance of the controllers are given in Table 3.

Figure 10 shows the responses obtained when the scheduling approach described above is applied to the controllers of Table 5 to control the $\mathrm{pH}$ neutralization process. For this process, windup does not present a problem, since all controllers are stable apart from the integrator, which can be taken as a common factor outside the switching mechanism.

As the nominal costs in Table 3 indicate, the performance achieved with the scheduled optimal controller is superior to that of both the optimal fixed controllers and the variable-gain controllers. In return, the implementation of scheduled control is more difficult. For the process studied, the scheduled controllers had to be designed for more robustness than the fixed and variablegain controllers in order to obtain closed-loop stability. In particular, attempts to achieve stability by scheduling locally optimal controllers designed for the uncertainty radius $\delta=0.5$ were not successful. 
Table 5. Parameters of locally optimal robust controllers at various operating points.

\begin{tabular}{|c|c|c|c|c|c|c|}
\hline $\mathbf{p H}$ & $\boldsymbol{g}_{\mathbf{1}}$ & $\boldsymbol{g}_{\mathbf{2}}$ & $\boldsymbol{g}_{\mathbf{3}}$ & \multicolumn{1}{c|}{$\boldsymbol{g}_{\mathbf{4}}$} & \multicolumn{1}{c|}{$\boldsymbol{g}_{\mathbf{5}}$} & $\boldsymbol{g}_{\mathbf{6}}$ \\
\hline 3 & $-1.098 \mathrm{e} 2$ & $-7.669 \mathrm{e} 4$ & $-2.589 \mathrm{e} 6$ & $-1.392 \mathrm{e} 7$ & $-3.131 \mathrm{e} 7$ & $-1.516 \mathrm{e} 7$ \\
\hline 4 & $-1.617 \mathrm{e}-1$ & $-8.646 \mathrm{e}-1$ & -2.421 & -2.428 & $-8.446 \mathrm{e}-1$ & $-7.075 \mathrm{e}-3$ \\
\hline 5 & $-6.043 \mathrm{e}-1$ & $-1.307 \mathrm{e} 1$ & $-8.281 \mathrm{e} 1$ & $-2.213 \mathrm{e} 2$ & $-2.273 \mathrm{e} 2$ & $-7.324 \mathrm{e}-1$ \\
\hline 6 & $-3.577 \mathrm{e} 1$ & $-9.273 \mathrm{e} 3$ & $-2.617 \mathrm{e} 5$ & $-1.669 \mathrm{e} 6$ & $-4.362 \mathrm{e} 6$ & $-3.887 \mathrm{e} 6$ \\
\hline 7 & $-1.049 \mathrm{e} 1$ & $-3.930 \mathrm{e} 2$ & $-2.712 \mathrm{e} 3$ & $-7.823 \mathrm{e} 3$ & $-8.620 \mathrm{e} 3$ & $-2.710 \mathrm{e} 3$ \\
\hline & $\boldsymbol{f}_{\mathbf{1}}$ & $\boldsymbol{f}_{\mathbf{2}}$ & $\boldsymbol{f}_{\mathbf{3}}$ & $\boldsymbol{f}_{\mathbf{4}}$ & $\boldsymbol{f}_{\mathbf{5}}$ & \\
\hline 3 & $1.010 \mathrm{e} 3$ & $3.191 \mathrm{e} 5$ & $4.918 \mathrm{e} 7$ & $3.106 \mathrm{e} 8$ & $2.208 \mathrm{e} 9$ & \\
\hline 4 & $3.276 \mathrm{e} 1$ & $2.245 \mathrm{e} 2$ & $1.210 \mathrm{e} 3$ & $1.086 \mathrm{e} 3$ & $1.119 \mathrm{e} 1$ & \\
\hline 5 & $1.169 \mathrm{e} 2$ & $3.586 \mathrm{e} 3$ & $2.245 \mathrm{e} 4$ & $1.493 \mathrm{e} 5$ & $8.778 \mathrm{e} 4$ & \\
\hline 6 & $5.296 \mathrm{e} 2$ & $1.481 \mathrm{e} 5$ & $1.290 \mathrm{e} 7$ & $8.662 \mathrm{e} 7$ & $6.207 \mathrm{e} 8$ & \\
\hline 7 & $1.390 \mathrm{e} 2$ & $1.497 \mathrm{e} 4$ & $1.082 \mathrm{e} 5$ & $8.003 \mathrm{e} 5$ & $6.418 \mathrm{e} 5$ & \\
\hline
\end{tabular}

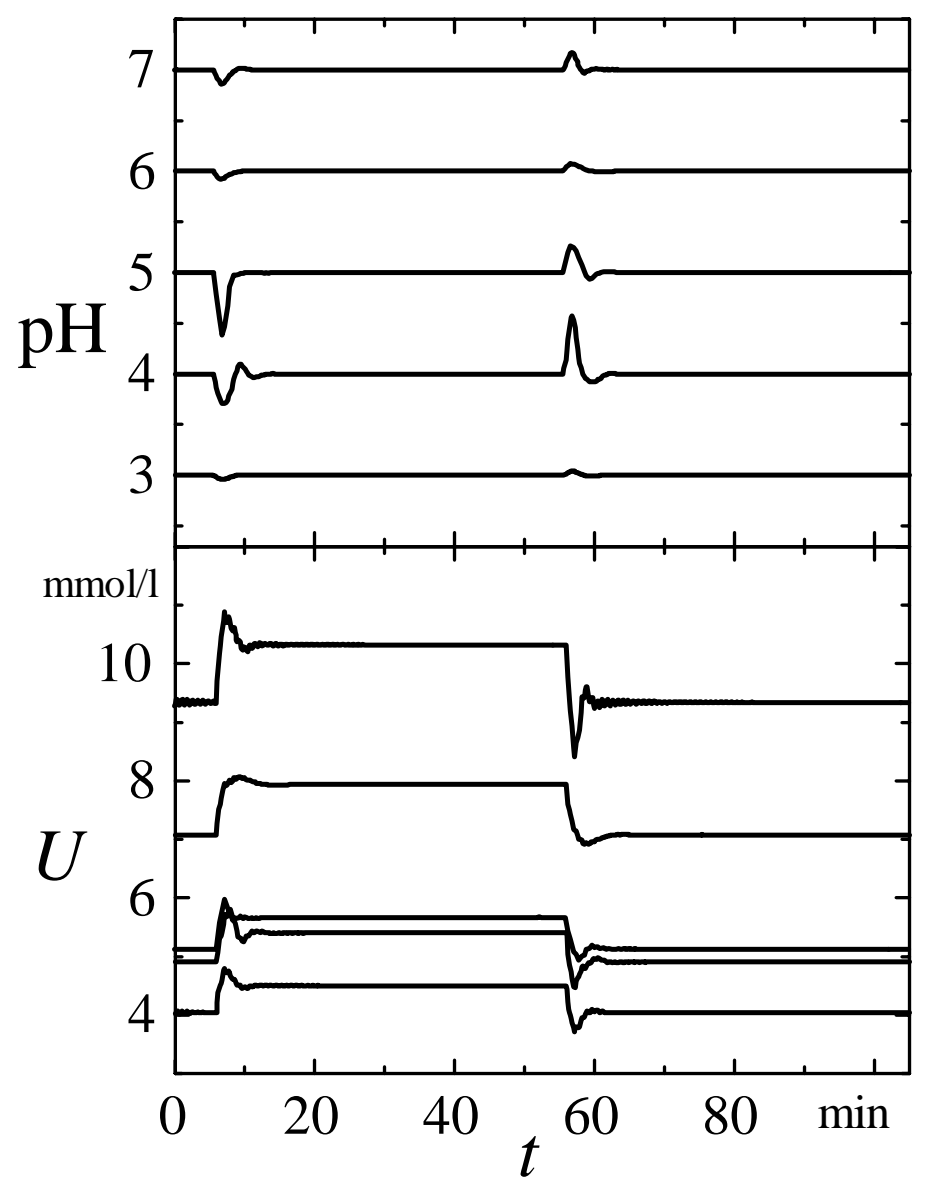

Figure 10. Simulation experiments using scheduled optimal robust controller. 


\subsection{PID control}

In this section, a conventional fixed PID controller using the modified ZieglerNichols method (Åström and Hägglund 1995) is designed for the pH process. The method is based on the idea of moving one point on the Nyquist curve, $r_{p} \mathrm{e}^{\mathrm{i}\left(\pi+\varphi_{p}\right)}$, to a desired position $r_{s} \mathrm{e}^{\mathrm{i}\left(\pi+\varphi_{s}\right)}$. The parameters specified by the user are the identification angle $\varphi_{p}$, and the parameters $r_{s}$ and $\varphi_{s}$ which define the desired point $S$. The specifications are given in terms of a phase margin $\varphi_{m}$ or an amplitude margin $A_{m}$. An amplitude margin design corresponds to $\varphi_{s}=0$ and $r_{s}=1 / A_{m}$, and a phase margin design corresponds to $r_{s}=1$ and $\varphi_{s}=\varphi_{m}$. The controller gain $K$, reset time $T_{i}$ and the derivative time $T_{d}$ are then given by (Åström and Hägglund 1995)

$$
\begin{aligned}
& K=\frac{r_{s} \cos \left(\varphi_{s}-\varphi_{p}\right)}{r_{p}} \\
& \omega T_{d}-\frac{1}{\omega T_{i}}=\tan \left(\varphi_{s}-\varphi_{p}\right)
\end{aligned}
$$

where $\omega$ is the critical frequency and where it is assumed that $T_{i}$ and $T_{d}$ have a given ratio, $T_{d}=\alpha T_{i}$, where $\alpha$ is often chosen as $\alpha=0.25$.

The PID controller for the process studied in this paper is tuned about the setpoint value $\mathrm{pH}=5$, where the process gain is very high. The parameters are chosen as: $\alpha=0.25, r_{s}=0.5, \varphi_{s}=\pi / 4$ and $\varphi_{p}=0$, which gives the controller parameters $K=6.37 \cdot 10^{-4}, T_{i}=2.57 \mathrm{~min}$ and $T_{d}=0.634 \mathrm{~min}$. 


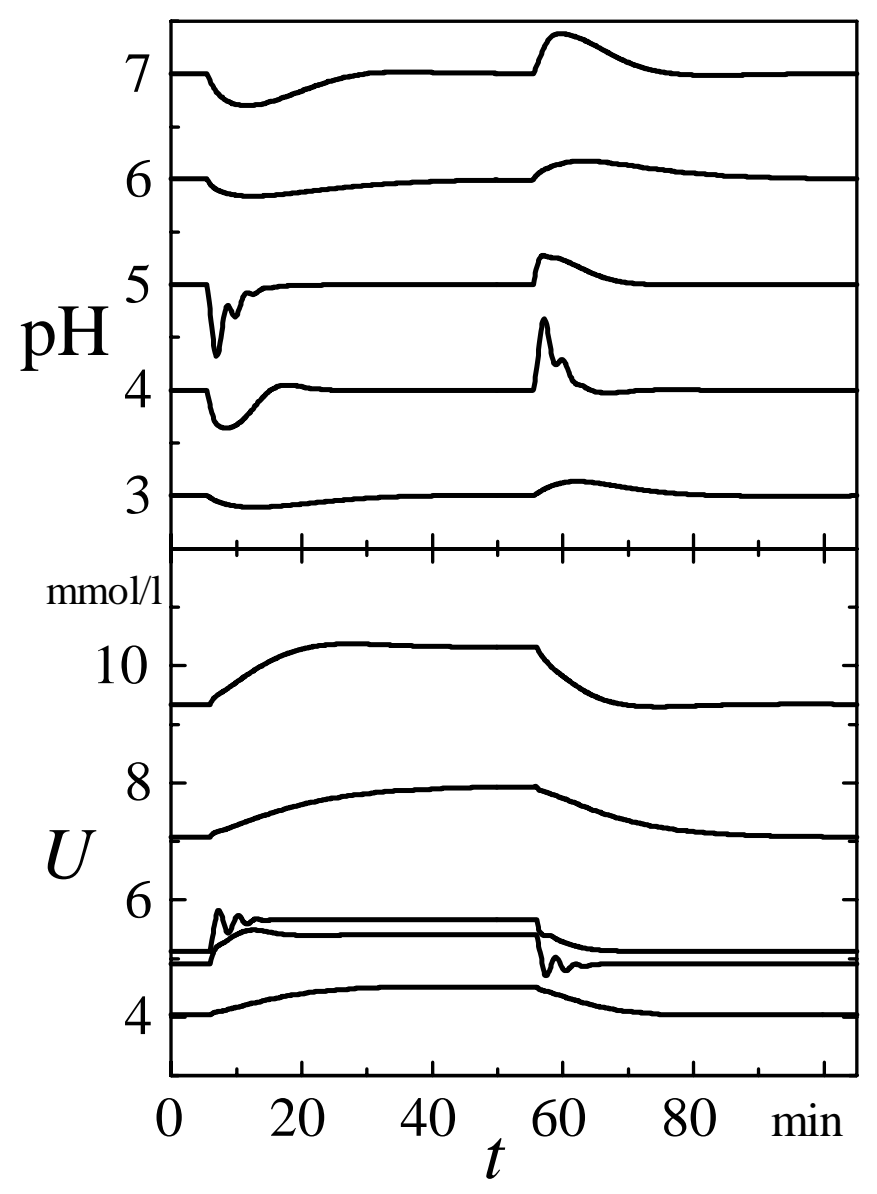

Figure 11. Simulation experiments using PID controller.

The nominal costs obtained with the PID controller at various operating points are given in Table 3. As expected, the performance is inferior at all operating points to the performance achieved with the optimally designed controllers. The simulated responses of the $\mathrm{pH}$ neutralization process in Figure 11 show that although the performance at the design point $\mathrm{pH} 5$ is acceptable, the controller response at the $\mathrm{pH}$ values 3, 6 and 7 is slow due to the small value of the process gain at these operating points. If the models valid at these points were used for tuning the PID controller, the closed loop would be unstable at $\mathrm{pH} 4$ and 5, where the process gain is large.

\section{Conclusion}

In this paper, a procedure for the design of optimal and robust controllers has been described and studied for a nonlinear chemical process. The design procedure is based on a multimodel process representation, in which the process is characterized in terms of a set of uncertain linear models. The characterization provides a quite general description of both parametric and 
norm-bounded uncertainties. The control problem is defined so as to achieve optimal LQ performance subject to robust stability for the set of uncertain models. This is equivalent to a multimodel mixed $H_{2} / H_{\infty}$ problem.

The multimodel mixed $H_{2} / H_{\infty}$ problem has been applied to design a linear controller which achieves optimal LQ performance and robust stability at a range of operating points of a nonlinear $\mathrm{pH}$ neutralization process. This is achieved by characterizing the nonlinear process in terms of a set of linearized models and their associated uncertainties.

For a strongly nonlinear process, it is clear that the global control performance which can be achieved by nonlinear control can be superior to the performance achievable by any linear controller. In this paper, the global performance of the multimodel mixed $\mathrm{H}_{2} / \mathrm{H}_{\infty}$ optimal controllers has been improved by controller scheduling methods. In particular, it has been shown that the design procedure can easily be modified in such a way that known variations of the locally valid stationary gain of the process are taken into account. The design leads to an easily implemented linear controller with variable gain. The case study has shown that in order to achieve good control performance by controller scheduling methods, it is essential that the controllers are properly implemented to satisfy the so-called linearization conditions.

The controller scheduling methods have the advantage of being fairly simple to implement, and the fact that linear controller design can be applied, which is in general simpler than nonlinear design techniques. The present study has shown that an approach using scheduling of linear controllers can perform quite well. An interesting topic for future studies would therefore be to compare the scheduled optimal controllers with optimal nonlinear controllers designed by nonlinear optimal and robust control theory.

The control problem studied in this paper has the property that the optimal controller lacks a closed-form solution. Therefore, the optimal controller must be computed by numerical optimization techniques. As the control problem is easy to formulate and is clearly well motivated in a number of applications, it seems worth while to study numerically efficient procedures for its solution. In this paper, the gradient expression of the associated costs have been derived, which can be applied in combination with general-purpose optimization routines. However, it is expected that numerically more efficient specialpurpose methods can be developed by exploiting the problem structure. This is left as a topic for future research. 


\section{Acknowledgment}

Financial support from the Academy of Finland and the Neste Foundation is gratefully acknowledged.

\section{References}

Anderson B.D.O. and J.B. Moore (1971). Linear Optimal Control. PrenticeHall.

Åström K.J. and T. Hägglund (1995). PID Controllers: Theory, Design, and Tuning. 2nd edition. Instrument Society of America.

Bernstein D.S. and W.M. Haddad (1989). LQG Control with an $H_{\infty}$ Performance Bound: A Riccati Equation Approach. IEEE Trans. Autom. Control 34, pp. 293-305.

Doyle J.C. (1982). Analysis of Feedback Systems with Structured Uncertainties. 'Proc. of the Inst. of Electr. Eng.' Part D, 133, pp. 45-56.

Doyle J.C., J.E. Wall and G. Stein (1982). Performance and Robustness Analysis for Structured Uncertainty. 'Proc. IEEE Conf. on Dec. and Contr.', Orlando, FL, pp. 629-636.

Gangsaas D., K.R. Bruce, J.D. Blight and U.-L. Ly (1986). Application of Modern Synthesis to Aircraft Control: Three Case Studies. IEEE Trans. Autom. Control 31, pp. 995-1041.

Green M. and D.J.N. Limebeer (1995). Linear Robust Control. Prentice-Hall.

Gustafsson T.K. and K.V. Waller (1983). Dynamic Modeling and Reaction Invariant Control of pH. Chem. Eng. Sci. 38, pp. 389-398.

Gustafsson T.K., B.O. Skrifvars, K.V. Sandström and K.V. Waller (1995). Modeling of pH for Control. Ind. Eng. Chem. Res. 34 pp. 820-827.

Kaminer I., A.M. Pascoal, P.P. Khargonekar and E.E. Coleman (1995). A Velocity Algorithm for the Implementation of Gain Scheduled Controllers. Automatica 31, pp. 1185-1191. 
Khargonekar P.P. and M.A. Rotea (1991). Mixed $\mathrm{H}_{2} / \mathrm{H}_{\infty}$ Control: A Convex Optimization Approach. IEEE Trans. Autom. Control 36, pp. 824-837.

Lawrence D.A. and W.J. Rugh (1995). Gain Scheduling Dynamic Linear Controllers for a Nonlinear Plant. Automatica 31, pp. 381-390.

MacMartin D.G., S.R. Hall and D.S. Bernstein (1991). Fixed-order Multimodel Estimation and Control. 'Proc. Amer. Contr. Conf.', Boston, MA, pp. 2113-2118.

Maciejowski J.M (1989). Multivariable Feedback Design. Addison Wesley Publishing Company, Inc.

Mäkilä P.M. (1991). Multiple Models, Multiplicative Noise and Linear Quadratic Control - Algorithm Aspects. Int. J. Control 54, pp. 921-941.

Miyazawa Y. (1990). Robust Flight Control System Design with Multiple Model Approach. 'Proc. AIAA Guid., Nav. and Control Conf.', Portland, OR, pp. 874-882.

Pensar J.A. (1996). Parametric Methods for Optimal and Robust Control. PhD thesis, Process Control Laboratory, Åbo Akademi, Åbo, Finland.

Pensar J.A. and H.T. Toivonen (1993). On the Design of fixed-structure $H_{\infty^{-}}$ optimal controllers. 'Proc. of the 32th Conf. Dec. Contr.', IEEE, San Antonio, TX, pp. 668-673.

Pensar J.A. and H.T. Toivonen (1994). A Multimodel Mixed $H_{2} / H_{\infty}$ Problem for Plants with Structured Uncertainty. 'Proc. ADCHEM', IFAC, Kyoto, Japan, pp. 289-294.

Ridgely D.B., C.P. Mracek and L. Valavani (1992a). Numerical Solutions to the General Mixed $\mathrm{H}_{2} / \mathrm{H}_{\infty}$ Optimization Problem. 'Proc. Amer. Contr. Conf.', Chicago, IL, pp. 1353-1357.

Ridgely D.B., L. Valavani, M. Dahleh and G. Stein (1992b). Solutions to the General Mixed $\mathrm{H}_{2} / \mathrm{H}_{\infty}$ Control Problem - Necessary Conditions for Optimality. 'Proc. Amer. Contr. Conf.', pp. 1348-1352. 
Sandelin P.M., H.T. Toivonen, M. Österås and K.V. Waller (1991). Robust Multiobjective Linear Quadratic Control of Distillation Using Low-order Controllers. Chem. Eng. Sci. 46, pp. 2815-2827.

Sandström K.V. and T.K. Gustafsson (1994). A Study of the Dynamics of Calcium-phosphate Precipitation in pH-control Systems. Report 94-5, Process Control Laboratory, Åbo Akademi, Åbo, Finland.

Skogestad S. and I. Postlethwaite (1996). Multivariable Feedback Control Analysis and Design. John Wiley \& Sons.

Toivonen H.T. and J. Tamminen (1990). Minimax Robust LQ Control of a Thermomechanical Pulping Plant. Automatica 26, pp. 347-351.

Vidyasagar M. (1985). Control System Synthesis: A Factorization Approach. MIT Press, Cambridge, Massachusetts.

Walton A-G (1967). The Formation and Properties of Precipitates. John Wiley \& Sons.

\section{Appendix A. Gradient of $J_{\infty}(P, F)$}

Consider the cost

$$
J_{\infty}(P, F):=\operatorname{tr}(Z U)
$$

where $Z$ is obtained from the Lyapunov equation

$$
A_{X}^{\mathrm{T}} Z+Z A_{X}+Q=0
$$

where

$$
A_{X}=A+B_{2} F C_{2}+\delta^{2}\left(B_{1}+B_{2} F D_{21}\right)\left(B_{1}+B_{2} F D_{21}\right)^{\mathrm{T}} X
$$

and $X$ is given by the Riccati equation

$$
\begin{aligned}
& \left(A+B_{2} F C_{2}\right)^{\mathrm{T}} X+X\left(A+B_{2} F C_{2}\right)+\delta^{2} X\left(B_{1}+B_{2} F D_{21}\right)\left(B_{1}+B_{2} F D_{21}\right)^{\mathrm{T}} X \\
& +\left(C_{1}+D_{12} F C_{2}\right)^{\mathrm{T}}\left(C_{1}+D_{12} F C_{2}\right)=0 .
\end{aligned}
$$


For the differentiation, consider the first-order expansion of $J_{\infty}(P, F)$ with respect to $F$ :

$$
\Delta J_{\infty}(P, F)=\operatorname{tr}\left\{\left(\frac{\partial J_{\infty}}{\partial F}\right)^{\mathrm{T}} \Delta F\right\}=\operatorname{tr}\left\{\left(\frac{\partial J_{\infty}}{\partial F}\right)_{X}^{\mathrm{T}} \Delta F\right\}+\operatorname{tr}\left\{\left(\frac{\partial J_{\infty}}{\partial X}\right)_{F}^{\mathrm{T}} \Delta X\right\} .
$$

From (A1) we obtain

$$
\operatorname{tr}\left\{\left(\frac{\partial J_{\infty}}{\partial F}\right)_{X}^{\mathrm{T}} \Delta F\right\}=\operatorname{tr}\left\{(\Delta Z)_{X} U\right\}
$$

where $(\Delta Z)_{X}$ denotes the first-order expansion of $Z ; Z(F+\Delta F)=Z(F)+(\Delta Z)_{X}+$ $o(\|\Delta F\|)$. From (A3) we have

$$
A_{X}^{\mathrm{T}}(\Delta Z)_{X}+(\Delta Z)_{X} A_{X}+M_{0}^{\mathrm{T}} Z+Z M_{0}=0
$$

where

$$
M_{0}=B_{2} \Delta F N^{\mathrm{T}} X+N \Delta F^{\mathrm{T}} B_{2}^{\mathrm{T}} X+B_{2} \Delta F C_{2}
$$

where

$$
N=\delta^{2}\left(B_{1}+B_{2} F D_{21}\right) D_{21}^{\mathrm{T}} .
$$

Introducing the Lyapunov equation

$$
A_{X} W+W A_{X}^{\mathrm{T}}+U=0
$$

into (A6), gives (using the symmetry $Z, W$ and $X$ )

$$
\begin{aligned}
& \operatorname{tr}\left\{\left(\frac{\partial J_{\infty}}{\partial F}\right)_{X}^{\mathrm{T}} \Delta F^{\mathrm{T}}\right\}=\operatorname{tr}\left\{\left((\Delta Z)_{X}\right) U\right\}=\operatorname{tr}\left\{W\left(M_{0}^{\mathrm{T}} Z+Z M_{0}\right)\right\} \\
& \quad=\operatorname{tr}\left\{\left(4 \delta^{2} D_{21} D_{21}^{\mathrm{T}} F^{\mathrm{T}} B_{2}^{\mathrm{T}} X+2 C_{2}+4 \delta^{2} D_{21} B_{1}^{\mathrm{T}} X\right) W Z B_{2} \Delta F\right\} .
\end{aligned}
$$

Similarly, we have 


$$
\operatorname{tr}\left\{\left(\frac{\partial J_{\infty}}{\partial X}\right)_{F}^{\mathrm{T}} \Delta X\right\}=\operatorname{tr}\left\{(\Delta Z)_{F} U\right\}
$$

where $(\Delta Z)_{F}$ is defined in analogy with $(\Delta Z)_{X}$, and is given by

$$
A_{X}^{\mathrm{T}}(\Delta Z)_{F}+(\Delta Z)_{F} A_{X}+\Delta X^{\mathrm{T}} M_{1}^{\mathrm{T}} Z+Z M_{1} \Delta X=0
$$

where

$$
M_{1}=\delta^{2}\left(B_{1}+B_{2} F D_{21}\right)\left(B_{1}+B_{2} F D_{21}\right)^{\mathrm{T}} .
$$

Introducing the Lyapunov equation (A10) into (A12) gives

$$
\begin{aligned}
& \operatorname{tr}\left\{\left(\frac{\partial J_{\infty}}{\partial X}\right)_{F}^{\mathrm{T}} \Delta X\right\}=\operatorname{tr}\left\{(\Delta Z)_{F} U\right\}=\operatorname{tr}\left\{2 W Z M_{1} \Delta X\right\} \\
& \quad=\operatorname{tr}\left\{2 \delta^{2} W Z\left(B_{1}+B_{2} F D_{21}\right)\left(B_{1}+B_{2} F D_{21}\right)^{\mathrm{T}} \Delta X\right\} .
\end{aligned}
$$

The Riccati equation (A4) gives

$$
\begin{aligned}
& A_{X}^{\mathrm{T}} \Delta X+\Delta X A_{X}+M^{\mathrm{T}} \Delta F C_{2}+C_{2}^{\mathrm{T}} \Delta F^{\mathrm{T}} M \\
& +X B_{2} \Delta F N^{\mathrm{T}} X+X N \Delta F^{\mathrm{T}} B_{2}^{\mathrm{T}} X=0
\end{aligned}
$$

where

$$
M=B_{2}^{\mathrm{T}} X+D_{12}^{\mathrm{T}}\left(C_{1}+D_{12} F C_{2}\right)
$$

Introducing the Lyapunov equation

$$
A_{X} Y+Y A_{X}^{\mathrm{T}}+2 \delta^{2} W Z\left(B_{1}+B_{2} F D_{21}\right)\left(B_{1}+B_{2} F D_{21}\right)^{\mathrm{T}}=0
$$

into (A15) gives

$$
\operatorname{tr}\left\{\left(\frac{\partial J_{\infty}}{\partial X}\right)_{F}^{\mathrm{T}} \Delta X\right\}=\operatorname{tr}\left\{\left[C_{2}\left(Y+Y^{\mathrm{T}}\right) M^{\mathrm{T}}+N^{\mathrm{T}} X\left(Y+Y^{\mathrm{T}}\right) X B_{2}\right] \Delta F\right\} .
$$

Finally, combining (A11) and (A19) gives 


$$
\begin{aligned}
& \Delta J_{\infty}(P, F)=\operatorname{tr}\left\{\left[\left(2 C_{2}+4 X N\right) W Z B_{2}+C_{2}\left(Y+Y^{\mathrm{T}}\right) M^{\mathrm{T}}\right.\right. \\
& \left.\left.\quad+N^{\mathrm{T}} X\left(Y+Y^{\mathrm{T}}\right) X B_{2}\right] \Delta F\right\}
\end{aligned}
$$

from which, by (A5), we have

$$
\begin{aligned}
\frac{\partial J_{\infty}}{\partial F}= & B_{2}^{\mathrm{T}} Z W\left(4 \delta^{2} X B_{2} F D_{21} D_{21}^{\mathrm{T}}+2 C_{2}^{\mathrm{T}}+4 \delta^{2} X B_{1} D_{21}^{\mathrm{T}}\right) \\
& +M\left(Y+Y^{\mathrm{T}}\right) C_{2}^{\mathrm{T}}+B_{2}^{\mathrm{T}} X\left(Y+Y^{\mathrm{T}}\right) X N \\
= & 2 B_{2}^{\mathrm{T}} Z W\left(C_{2}^{\mathrm{T}}+2 X N\right)+M\left(Y+Y^{\mathrm{T}}\right) C_{2}^{\mathrm{T}}+B_{2}^{\mathrm{T}} X\left(Y+Y^{\mathrm{T}}\right) X N . \quad(\text { A2 } 1)
\end{aligned}
$$

